\title{
A Phase-Field Method for 3D Simulation of Two-Phase Heat Transfer
}

\author{
X. Zheng, H. Babaee, S. Dong, C. Chryssostomidis, G.E. Karniadakis* \\ ${ }^{a}$ Department of Mechanical Engineering, Massachusetts Institute of Technology \\ ${ }^{b}$ Department of Mechanical Engineering, Massachusetts Institute of Technology, United States \\ ${ }^{c}$ Department of Mathematics, Purdue University \\ ${ }^{d}$ Department of Mechanical Engineering, Massachusetts Institute of Technology, United States \\ ${ }^{e}$ Division of Applied Mathematics, Brown University
}

\begin{abstract}
We formulate new multi-phase convective heat transfer equations by combining the threedimensional (3D) Navier-Stokes equations, the energy equation and the Cahn-Hilliard equation for the phase field variable $\phi(\mathbf{x}, t)$. The density, viscosity, heat capacity and conductivity are functions of $\phi(\mathbf{x}, t)$. The equations are solved in time with a splitting scheme that decouples the flow and temperature variables, yielding time-independent coefficient matrices after discretization, which can be computed during pre-processing. Here, a spectral element method is employed for spatial discretization but any other Eulerian grid discretization scheme is also suitable. We test the new method in several 3D benchmark problems for convergence in time/space including a conjugate heat transfer problem and also for a realistic transient cooling of a 3D hot object in a cavity with a moving air-water interface. These applications demonstrate the efficiency of the new method in simulating 3D multi-phase convective heat transfer on stationary grids, different modes of heat transfer (e.g convection/conduction), as well as its robustness in handling different fluids with large contrasts

\footnotetext{
${ }^{*}$ Corresponding author

Email address: george_karniadakis@brown.edu 617-253-4335 (G.E. Karniadakis)
} 
in physical properties.

Keywords: spectral element, non-moving grid, Cahn-Hilliard equation, large thermal conductivity ratio

\section{Introduction}

Heat transfer in conjunction with multi-phase flow is ubiquitous in many engineering and scientific applications involving phase change, distillation, extraction, absorption and drying. The modeling and numerical simulation of multi-phase flow systems have therefore been the subject of numerous theoretical and computational studies $[1,2,3,4,5]$.

The common modeling approaches can be broadly divided into two basic types of sharpand diffuse-interface models. The sharp-interface models assume a zero-thickness layer that separates the two fluids. This layer is endowed with properties such as surface tension, and matching boundary conditions are imposed on either side of this surface. For numerical simulation of two-phase flow systems with sharp-interface models, moving-grid methods are commonly used with conformal elements on either side of the interface [6, 7]. However, the possibility of mesh entanglement restricts the moving-grid approaches to cases with mild deformation of the interface. Such a limitation forbids any morphological changes, unless a new grid is generated "on-the-fly", which significantly hampers the efficiency of the method.

More recently, Smoothed Particle Hydrodynamics (SPH) has also been successfully used in modeling two-phase flow system. The SPH model employs a purely Lagrangian viewpoint in which the particles are moving as interpolation points, and the inner-particle forces (viscous, pressure, etc) are calculated by smoothing the properties of its neighboring particles while satisfying the Navier-Stokes equations. This approach provides a suitable framework 
for tracking different phases, in multi-phase flow systems. For the application of SPH method in multi-phase flow systems see for instance $[8,9,10]$.

The diffuse-interface models, however, assume a finite-thickness layer between the two phases. The interfacial tension between the two fluids spreads over this narrow layer. This approach yields a unified set of governing equations for two phases, instead of formulating the flow in two separate domains. Numerical methods such as Volume-of-Fluid (VOF) [11, 12, $13,14]$ and level-set $[14,15,16]$ have been successfully employed by utilizing diffuse-interface models to simulate two-phase systems.

From the modeling perspective, the energy-based variational framework of phase-field formulation makes it a thermodynamically-consistent and physically attractive approach to modele multi-phase flow systems (see for instance [17]). Unlike the level-set model, where an artificial smoothing function is prescribed for the interface, the Cahn-Hillard model describes the interface by a mixing energy, and in that sense, the phase-field model can be viewed as a physically motivated level-set method. The energy-based description of phase-field model can also allow complex rheology of non-Newtonian fluids to be easily incorporated into the formulation [18]. On the other hand, from the numerical viewpoint, the phase-field method provides a single set of partial differential equations for two phases that can be discretized on a fixed grid in an Eulerian framework. It can also handle morphological changes such as breakup, coalescence and reconnection, which extends the application of the method to complex two-phase flow systems. An example of a 2D simulation of a cold water jet impinging on a hot air-water interface is shown in Fig. 1. Initially we have cold water $\left(10^{\circ} \mathrm{C}\right)$ issuing from the middle of the upper wall. Hot water $\left(50^{\circ} \mathrm{C}\right)$ fills up the bottom half of the domain. There are two outlets at the two corners of the upper wall. Upper and lower walls assume 
adiabatic temperature boundary conditions and periodic in horizontal direction. As shown in Fig. 1(b), blue color represents water, which corresponds to $\phi(\mathbf{x}, t)=-1$, while white color represents air, which corresponds to $\phi(\mathbf{x}, t)=1$. The interface between water and air is provided by the solution of $\phi(\mathbf{x}, t)$ from the Cahn-Hilliard equation but not tracked, which is different from the interface tracking/capturing techniques.

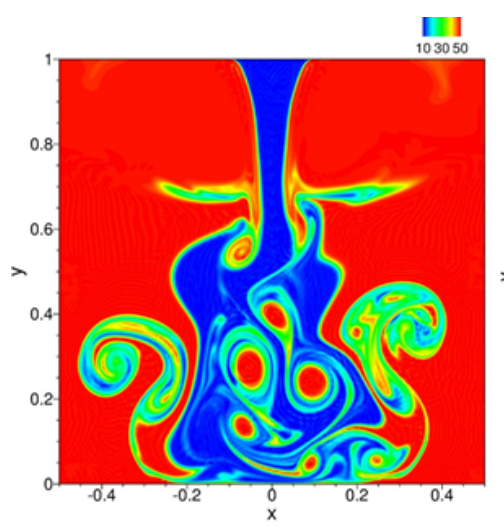

(a)

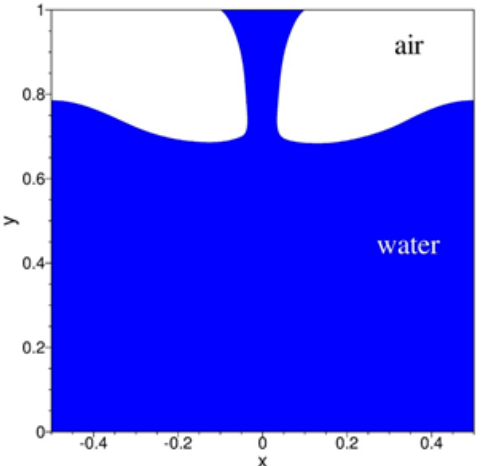

(b)

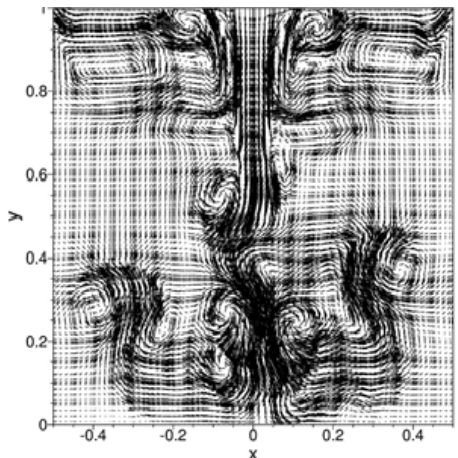

(c)

Figure 1: Cold water jet $10^{\circ} \mathrm{C}$ issuing from the middle of the upper wall into a hot air-water pool $50^{\circ} \mathrm{C}$ : (a) temperature snapshot; (b) air-water interface snapshot in terms of the phase field. Initially $\phi(\mathbf{x}, t)=1$ for air and $\phi(\mathrm{x}, t)=-1$ for water; (c) velocity snapshot.

However, there are several challenges in the numerical simulation of the Cahn-Hillard equation coupled with convective heat transfer equations that must be remedied for the model to be used in realistic applications. In cases with large thermal conductivity and density ratios, the discretization of the phase-field formulation combined with heat transfer equations leads to highly stiff discrete systems, causing numerical stability issues. The cases with large ratios of physical properties are plentiful in realistic applications such as water-air systems or most systems where phase change is involved. On the other hand, the phase-field 
formulation renders physical properties (such as density, thermal conductivity, viscosity, ...) as time-dependent variables through their dependence on the phase field $\phi(\mathbf{x}, t)$. The timedependence of these properties makes the coefficient matrices time-dependent accordingly, requiring an expensive computing/assembling of these matrices at each time step, and thus significantly hampering the numerical efficiency of the algorithm. Moreover, the convective heat transfer equations combined with Cahn-Hillard equation form a fully coupled system of partial differential equations. Hence, a de-coupling strategy is very desirable in order to avoid the high computational cost incurred by solving such a coupled system of equations.

Among the existing methods of discretizing the phase-field formulation for convective heat transfer problems, the spectral/hp element method, in particular, is very promising [19]. The smooth transition of phase field and physical properties between the two phases makes this method compliant with sufficient regularity required in spectral-type element discretizations. The low dispersion error of spectral/hp discretization compared to loworder methods is also attractive in convection-dominated problems. For more details on spectral/hp element method see reference [20]. However, any other finite difference, finite volume or finite element method can be combined with the approach proposed here.

In this paper we present an efficient numerical algorithm for discretizing multi-phase convective heat transfer equations. We employ a splitting scheme as a decoupling strategy to efficiently solve the system of PDEs obtained from phase-field formulation. Our method results in time-independent coefficient matrices that can be pre-computed during the pre-processing. We verify our method by comparing the numerical results with analytical solutions. We also demonstrate the capability of our method by simulating the flow of a water-air system with density ratio of 1000 around a hot object. 
This paper is organized as follows. In the next section we develop the numerical algorithm for discretizing multi-phase convective heat transfer equation using the phase-field methodology. In the third section, we demonstrate the spatial and time convergence of the proposed method. In the fourth section, we verify the accuracy of the numerical temperature field with an exact solution for a two-phase flow convection problem in a pipe and a conjugate heat transfer problem in a channel. In the last section we show the results of simulation of a transient cooling of a hot object immersed in water-air flow with a moving interface.

\section{Numerical method}

\subsection{Governing equations and boundary conditions}

Let $\Omega$ denote an open bounded domain in two or three dimensions (2-D or $3-\mathrm{D})$, and let $\partial \Omega$ denote its boundary. We consider a mixture of two immiscible incompressible fluids, with different viscous and thermal properties, contained in $\Omega$. Let $\rho_{1}$ and $\rho_{2}$, respectively, denote the densities of the two fluids, $\mu_{1}$ and $\mu_{2}$ denote their dynamic viscosities, $c_{1}$ and $c_{2}$ denote their specific heat coefficients, and $k_{1}$ and $k_{2}$ denote their thermal conductivities. We assume that there is no phase change in the system. This two-phase system can be described by the following system of equations:

$$
\begin{aligned}
& \rho\left(\frac{\partial \mathbf{u}}{\partial t}+\mathbf{u} \cdot \nabla \mathbf{u}\right)=-\nabla p+\nabla \cdot\left[\mu\left(\nabla \mathbf{u}+\nabla \mathbf{u}^{T}\right)\right]-\lambda \nabla \cdot(\nabla \phi \nabla \phi)+\mathbf{f}(\mathbf{x}, t), \\
& \nabla \cdot \mathbf{u}=0 \\
& \frac{\partial \phi}{\partial t}+\mathbf{u} \cdot \nabla \phi=-\lambda \gamma_{1} \nabla^{2}\left[\nabla^{2} \phi-h(\phi)\right]+g(\mathbf{x}, t), \\
& \rho c\left(\frac{\partial T}{\partial t}+\mathbf{u} \cdot \nabla T\right)=\nabla \cdot(k \nabla T)+q_{s}(\mathbf{x}, t) .
\end{aligned}
$$


In the above equations, $\mathbf{u}(\mathbf{x}, t)$ is velocity, $p(\mathbf{x}, t)$ is pressure, $T(\mathbf{x}, t)$ is temperature, $\mathbf{f}(\mathbf{x}, t)$ is a body force (such as gravity), and $q_{s}(\mathbf{x}, t)$ is a heat source, where $t$ is time and $\mathbf{x}$ is the spatial coordinate; $\phi(\mathbf{x}, t)$ is the phase field function, where $-1 \leqslant \phi \leqslant 1$. The flow regions with $\phi=1$ and $\phi=-1$, respectively, representing the first and the second fluids. The iso-surface $\phi(\mathbf{x}, t)=0$ marks the interface between the two fluids at time $t$, while $h(\phi)$ is given by $h(\phi)=\frac{1}{\eta^{2}} \phi\left(\phi^{2}-1\right)$, and $\eta$ is a characteristic length scale of the interface thickness. Also, $\lambda$ is the mixing energy density coefficient and is related to the surface tension $\sigma$ by $\lambda=\frac{3}{2 \sqrt{2}} \sigma \eta[18]$. There is a diffusion term $\left(\nabla^{2} \phi-h(\phi)\right)$ on the right hand side of Eq. (1c), which is different from corresponding terms in the Volume-of-Fluid (VOF) or the level set functions. This diffusion term $\left(\nabla^{2} \phi-h(\phi)\right)$ is the gradient of the mixing energy density $\mathrm{W}$, where $W=\int_{\Omega}\left(\lambda / 2|\nabla \phi|^{2}+\lambda / 4 / \eta^{2}\left(\phi^{2}-1\right)^{2}\right) d \Omega$. The first term of the integrand tends to mix the two fluids so that the gradient will approach zero when energy is minimized; the second term (double-well potential) of the integrand tends to seperate the two fluids. The interplay of these two tendencies will determine the dynamic profile of the interface, see [18]. In the current paper we assume that the surface tension $\sigma$ is not affected by the temperature and $\gamma_{1}$ is the mobility of the interface, and is assumed to be constant; $g(\mathbf{x}, t)$ in Eq. (1c) is a prescribed source term, which will be set to $g(\mathbf{x}, t)=0$ in present simulations. The density $\rho$, dynamic viscosity $\mu$, specific heat $c$, and the thermal conductivity $k$, are related to the 
phase field function by:

$$
\left\{\begin{array}{l}
\rho(\phi)=\frac{1}{2}\left(\rho_{1}+\rho_{2}\right)+\frac{1}{2}\left(\rho_{1}-\rho_{2}\right) \phi \\
\mu(\phi)=\frac{1}{2}\left(\mu_{1}+\mu_{2}\right)+\frac{1}{2}\left(\mu_{1}-\mu_{2}\right) \phi \\
c(\phi)=\frac{1}{2}\left(c_{1}+c_{2}\right)+\frac{1}{2}\left(c_{1}-c_{2}\right) \phi \\
k(\phi)=\frac{1}{2}\left(k_{1}+k_{2}\right)+\frac{1}{2}\left(k_{1}-k_{2}\right) \phi
\end{array}\right.
$$

Consequently, the density, dynamic viscosity, specific heat, and thermal conductivity in Eqs. (1a) and (1d) are all time-dependent field variables. In the current paper, we assume that the individual densities $\left(\rho_{1}\right.$ and $\left.\rho_{2}\right)$, dynamic viscosities $\left(\mu_{1}\right.$ and $\left.\mu_{2}\right)$, specific heat $\left(c_{1}\right.$ and $\left.c_{2}\right)$, and thermal conductivities $\left(k_{1}\right.$ and $\left.k_{2}\right)$ of the two fluids are all constants and do not depend on the temperature field $T$, but this assumption can be removed in future formulations.

In the above set of equations, (1a) is the augmented Navier-Stokes equation, involving a variable density and a variable dynamic viscosity, in which the term $\lambda \nabla \cdot(\nabla \phi \nabla \phi)$ represents the surface tension effect. Eq. (1c) (with $g=0$ ) is the Cahn-Hilliard equation. In the temperature equation $(1 \mathrm{~d})$, we have assumed that the heat generated due to the fluid viscosity is negligible.

One can observe that the velocity, pressure, and the phase field functions are fully coupled with one another. The temperature field is affected by the velocity and the phase field function. On the other hand, under the assumption that the surface tension and the individual physical parameters (density, dynamic viscosity, specific heat and thermal conductivity) of the two fluids are temperature-independent, the temperature field does not influence the flow fields (velocity, pressure and phase field function). We consider this "one-way" coupled configuration with respect to the temperature in the current paper. Our emphasis is on devising efficient algorithms to overcome the computational challenges posed by the time- 
dependent thermal conductivity, time-dependent specific heat, time-dependent density, and time-dependent viscosity.

The system of equations, (1a)-(1d), is supplemented by appropriate boundary conditions for the velocity, phase field function, and temperature. For the velocity, we assume a Dirichlet condition on the boundary and Neumann at the outflow,

$$
\mathbf{u}=\mathbf{w}(\mathbf{x}, t), \quad \text { on } \partial \Omega
$$

where $\mathbf{w}$ is the boundary velocity.

For the phase field function, we assume the static contact-angle condition (see [19]) on the boundary,

$$
\begin{array}{ll}
\mathbf{n} \cdot \nabla\left[\nabla^{2} \phi-h(\phi)\right]=g_{a}(\mathbf{x}, t), & \text { on } \partial \Omega, \\
\mathbf{n} \cdot \nabla \phi=-\frac{1}{\lambda} f_{w}^{\prime}(\phi)+g_{b}(\mathbf{x}, t), & \text { on } \partial \Omega,
\end{array}
$$

where $g_{a}$ and $g_{b}$ are prescribed functions on $\partial \Omega$, and will be set to $g_{a}=g_{b}=0$ in present simulations. Also, $f_{w}$ is the fluid-solid interfacial tension function and is given by [19],

$$
f_{w}(\phi)=\sigma \cos \theta_{s} \frac{\phi\left(\phi^{2}-3\right)}{4}+\frac{1}{2}\left(\sigma_{w 1}+\sigma_{w 2}\right)
$$

where $\theta_{s}$ is the equilibrium (static) contact angle between the fluid interface and the solid wall measured on the side of the first fluid, and the constant $\sigma_{w 1}$ is the fluid one-solid interfacial tension, while the constant $\sigma_{w 2}$ is the fluid two-solid interfacial tension.

For the temperature, we consider several types of boundary conditions. We assume that $\partial \Omega=\partial \Omega_{d}^{T} \cup \partial \Omega_{n}^{T} \cup \partial \Omega_{r}^{T}$, where $\partial \Omega_{d}^{T}, \partial \Omega_{n}^{T}$ and $\partial \Omega_{r}^{T}$, respectively, corresponding to Dirichlet, Neumann and Robin type boundaries. Specifically, on the Dirichlet boundary we impose the 
temperature condition

$$
T=T_{d}(\mathbf{x}, t), \quad \text { on } \partial \Omega_{d}^{T}
$$

where $T_{d}$ is the boundary tempeature. On the Neumann boundary we impose the heat flux condition

$$
-k(\phi) \mathbf{n} \cdot \nabla T=h_{n}(\mathbf{x}, t), \quad \text { on } \partial \Omega_{n}^{T}
$$

where $h_{n}$ is the given heat flux function on $\partial \Omega_{n}^{T}$. On the Robin boundary we impose a convective heat transfer condition

$$
-k(\phi) \mathbf{n} \cdot \nabla T=h_{c}\left(T-T_{a}\right)+q_{r}(\mathbf{x}, t), \quad \text { on } \partial \Omega_{r}^{T}
$$

where $h_{c}(\mathbf{x}, t)>0$ is the convective heat transfer coefficient and is finite, $T_{a}$ is a reference temperature, and $q_{r}$ is a prescribed function on $\partial \Omega_{r}^{T}$ and will be set to $q_{r}=0$ in actual simulations. We note that the variable thermal conductivity $k(\phi)$ is involved in the Neumann and Robin type boundary conditions.

The Eqs. (1a)-(1d), the boundary conditions (3)-(4b) and (6)-(8), and appropriate initial conditions for the velocity, phase field function and temperature, together constitute the system that needs to be solved in two-phase heat transfer simulations.

When solving this system, the primary numerical challenge is posed by the variable density, variable dynamic viscosity, variable specific heat, and variable thermal conductivity in the governing equations as well as in the boundary conditions. With usual algorithmic formulations, these variable physical parameters result in variable (time-dependent) coefficient matrices for the linear algebraic systems after discretization for different flow variables. Consequently, these coefficient matrices need to be re-computed every time step. This creates a 
severe bottleneck in performance because of the cost associated with coefficient matrix computation, which severely hampers long-time simulations of two-phase heat transfer problems.

\subsection{Algorithm formulation and implementation}

In this section we present an efficient algorithm that can overcome the challenge caused by the variable coefficient matrices associated with variable physical parameters. We first develop an algorithm for the two-phase temperature equation together with temperature boundary conditions, which overcomes the aforementioned difficulties. Then, we combine this algorithm together with a scheme for the two-phase flow equations developed recently in [21] (summarized in Appendix A) to form an efficient method for simulating two-phase heat transfer problems.

We first consider the two-phase temperature equation (1d) together with the boundary conditions (6)-(8), assuming that the velocity $\mathbf{u}$ and the phase field function $\phi$ are both known. We rewrite Eq. (1d) as

$$
\frac{\partial T}{\partial t}+\mathbf{u} \cdot \nabla T=\frac{k}{\rho c} \nabla^{2} T+\frac{1}{\rho c} \nabla k \cdot \nabla T+\frac{1}{\rho c} q_{s}
$$

Let $\chi^{n}$ denote $\chi$ at time step $n$, where $\chi$ is a generic variable. Given $T^{n}$, we compute $T^{n+1}$ as follows:

$$
\begin{gathered}
\frac{\gamma_{0} T^{n+1}-\hat{T}}{\Delta t}+\mathbf{u}^{n+1} \cdot \nabla T^{*, n+1}=\alpha_{m} \nabla^{2} T^{n+1}+\left(\frac{k^{n+1}}{\rho^{n+1} c^{n+1}}-\alpha_{m}\right) \nabla^{2} T^{n+1} \\
+\frac{1}{\rho^{n+1} c^{n+1}} \nabla k^{n+1} \cdot \nabla T^{*, n+1}+\frac{1}{\rho^{n+1} c^{n+1}} q_{s}^{n+1}, \\
T^{n+1}=T_{d}^{n+1}, \quad \text { on } \partial \Omega_{d}^{T},
\end{gathered}
$$




$$
\begin{gathered}
-k^{n+1} \mathbf{n} \cdot T^{n+1}=h_{n}^{n+1}, \quad \text { on } \partial \Omega_{n}^{T}, \\
-k^{n+1} \mathbf{n} \cdot \nabla T^{n+1}=h_{c}^{n+1}\left(T^{n+1}-T_{a}\right)+q_{r}^{n+1}, \quad \text { on } \partial \Omega_{r}^{T} .
\end{gathered}
$$

In the above equations, $\Delta t$ is the time step size, and $\mathbf{u}^{n+1}$ and $\phi^{n+1}$ are known from the previous stage in the same time step. $\rho^{n+1}, c^{n+1}$ and $k^{n+1}$ can be computed based on Eq. (2), and are therefore also known. $T^{*, n+1}$ is a $J$-th order ( $\mathrm{J}$ controls the order of temporal accuracy, $J=1$ or 2) explicit approximation of $T^{n+1}$, which is given by

$$
T^{*, n+1}= \begin{cases}T^{n}, & \text { if } J=1, \\ 2 T^{n}-T^{n-1}, & \text { if } J=2 .\end{cases}
$$

The expression $\frac{1}{\Delta t}\left(\gamma_{0} T^{n+1}-\hat{T}\right)$ is an approximation of $\frac{\partial T}{\partial t}$ at time step $(n+1)$ with the $J$-th order backward differentiation formula [21], where

$$
\gamma_{0}=\left\{\begin{array}{ll}
1, & \text { if } J=1, \\
\frac{3}{2}, & \text { if } J=2,
\end{array} \quad \hat{T}= \begin{cases}T^{n}, & \text { if } J=1, \\
2 T^{n}-\frac{1}{2} T^{n-1}, & \text { if } J=2 .\end{cases}\right.
$$

For stability reasons, we choose $\alpha_{m}$ to be a positive constant satisfying the condition $\alpha_{m} \geqslant$ $\frac{1}{2} \frac{\max \left(k_{1}, k_{2}\right)}{\min \left(\rho_{1}, \rho_{2}\right) \cdot \min \left(c_{1}, c_{2}\right)}$.

Specifically, in Eq. (10a), to deal with the variable coefficient term $\frac{k}{\rho c} \nabla^{2} T$, we have reformulated it into two terms. The first constant-coefficient term, $\alpha_{m} \nabla^{2} T$, is treated implicitly. The second correction term, $\left(\frac{k}{\rho c}-\alpha_{m}\right) \nabla^{2} T$, is also treated implicitly at this point, but will be further approximated in subsequent developments. This type of strategy for splitting the variable coefficient term can be traced to an idea suggested in [22]; see also its use for Navier-Stokes equations [21, 23]. 
Let $H_{T 0}^{1}(\Omega)=\left\{v \in H^{1}(\Omega):\left.v\right|_{\partial \Omega_{d}^{T}}=0\right\}$, and $\varphi \in H_{T 0}^{1}(\partial \Omega)$ denote the test function. We take the $L^{2}$ inner product between Eq. (10a) and $\varphi$, integrate by part, and we obtain the weak form

$$
\begin{aligned}
& \int_{\Omega} \nabla T^{n+1} \cdot \nabla \varphi+\frac{\gamma_{0}}{\alpha_{m} \Delta t} \int_{\Omega} T^{n+1} \varphi=\frac{1}{\alpha_{m}} \int_{\Omega} R \varphi-\frac{1}{\alpha_{m}} \int_{\Omega}\left(\frac{k^{n+1}}{\rho^{n+1} c^{n+1}}-\alpha_{m}\right) \nabla T^{n+1} \cdot \nabla \varphi \\
& -\frac{1}{\alpha_{m}} \int_{\Omega} \nabla\left(\frac{k^{n+1}}{\rho^{n+1} c^{n+1}}\right) \cdot \nabla T^{n+1} \varphi+\frac{1}{\alpha_{m}} \int_{\partial \Omega} \frac{k^{n+1}}{\rho^{n+1} c^{n+1}} \mathbf{n} \cdot \nabla T^{n+1} \varphi, \quad \forall \varphi \in H_{T 0}^{1}(\Omega), \quad(13)
\end{aligned}
$$

where

$$
R=\frac{1}{\rho^{n+1} c^{n+1}} q_{s}^{n+1}-\mathbf{u}^{n+1} \cdot \nabla T^{*, n+1}+\frac{\hat{T}}{\Delta t}+\frac{1}{\rho^{n+1} c^{n+1}} \nabla k^{n+1} \cdot \nabla T^{*, n+1},
$$

and we have used the relation ( $B$ denoting a scalar function)

$$
B \nabla^{2} T \varphi=\nabla \cdot(B \nabla T \varphi)-B \nabla T \cdot \nabla \varphi-\nabla B \cdot \nabla T \varphi
$$

We next approximate explicitly the term $\nabla T^{n+1}$ in the volume integrals on the right hand side (RHS) of (13), and obtain the final weak form for $T^{n+1}$,

$$
\begin{gathered}
\int_{\Omega} \nabla T^{n+1} \cdot \nabla \varphi+\frac{\gamma_{0}}{\alpha_{m} \Delta t} \int_{\Omega} T^{n+1} \varphi+\frac{\beta_{m}}{\alpha_{m}} \int_{\partial \Omega_{r}^{T}} T^{n+1} \varphi=\frac{1}{\alpha_{m}} \int_{\Omega} R \varphi \\
-\frac{1}{\alpha_{m}} \int_{\Omega}\left(\frac{k^{n+1}}{\rho^{n+1} c^{n+1}}-\alpha_{m}\right) \nabla T^{*, n+1} \cdot \nabla \varphi \\
\quad-\frac{1}{\alpha_{m}} \int_{\Omega} \nabla\left(\frac{k^{n+1}}{\rho^{n+1} c^{n+1}}\right) \cdot \nabla T^{*, n+1} \varphi-\frac{1}{\alpha_{m}} \int_{\partial \Omega_{n}^{T}} \frac{1}{\rho^{n+1} c^{n+1}} h_{n}^{n+1} \varphi \\
+\frac{1}{\alpha_{m}} \int_{\partial \Omega_{r}^{T}}\left[\left(\beta_{m}-\frac{h_{c}^{n+1}}{\rho^{n+1} c^{n+1}}\right) T^{*, n+1}+\frac{1}{\rho^{n+1} c^{n+1}}\left(h_{c}^{n+1} T_{a}+q_{r}^{n+1}\right)\right] \varphi, \quad \forall \varphi \in H_{T 0}^{1}(\Omega),
\end{gathered}
$$

where $\beta_{m}$ is a chosen constant satisfying condition $\beta_{m} \geqslant \frac{h_{c, \max }}{\min \left(\rho_{1}, \rho_{2}\right) \cdot \min \left(c_{1}, c_{2}\right)}$ (for stability as before), and we have used Eqs. (10c) and (10d) and the following reformulation on the Robin boundary $\partial \Omega_{r}^{T}$

$$
\int_{\partial \Omega_{r}^{T}} \frac{h_{c}^{n+1}}{\rho^{n+1} c^{n+1}} T^{n+1} \varphi \approx \beta_{m} \int_{\partial \Omega_{r}^{T}} T^{n+1} \varphi+\int_{\partial \Omega_{r}^{T}}\left(\frac{h_{c}^{n+1}}{\rho^{n+1} c^{n+1}}-\beta_{m}\right) T^{*, n+1} \varphi
$$


as well as the fact that $\int_{\partial \Omega_{d}^{T}} \mathbf{n} \cdot \nabla T^{n+1} \varphi=0$ because $\varphi \in H_{T 0}^{1}(\Omega)$.

Our algorithm for computing the temperature $T^{n+1}$ therefore consists of solving Eq. (16) for $T^{n+1}$ together with the Dirichlet condition (10b) on $\partial \Omega_{d}^{T}$. Eq. (16) is in weak form and involves no derivatives of order two or higher. Therefore, it can be readily discretized in space with $C^{0}$ spectral elements or finite elements. We employ $C^{0}$ spectral elements for spatial discretizations [20] in the current paper but any standard finite element or finite volume or finite difference method would be suitable.

One can observe that Eq. (16) involves a constant and time-independent coefficient matrix for the linear algebraic system for $T^{n+1}$ after spatial discretization, even though the original governing equation (1d) and the boundary conditon (8) involve variable physical parameters $\rho(\phi), c(\phi)$ and $k(\phi)$. The coefficient matrix can therefore be pre-computed during pre-processing, and stored for time marching in subsequent time steps. This is a prominent feature of our algorithm, which makes our approach very efficient. Our algorithm effectively overcomes the performance bottleneck of the two-phase temperature equation caused by variable coefficient matrices associated with variable specific heat, variable thermal conductivity, and variable density.

We next combine the algorithm discussed above for the two-phase temperature equation, and an algorithm developed previously in [21] for the two-phase flow equations, to arrive at an efficient method for simulating two-phase heat transfer problems. For the sake of completeness, in Appendix A we have summarized our algorithm for the two-phase flow equations (1a)-(1c), together with the boundary conditions (3), (4a) and (4b).

Given $\left(\mathbf{u}^{n}, P^{n}, \phi^{n}, T^{n}\right)$, our overall algorithm for two-phase heat transfer problems consists of the following steps within a time step $\Delta t$ : 
1. Solve Eq. (31) (see Appendix A) for $\psi^{n+1}$.

2. Solve Eq. (32) (see Appendix A) for $\phi^{n+1}$.

3. Compute $\rho^{n+1}, \mu^{n+1}, c^{n+1}$ and $k^{n+1}$ from Eq. (2), or at very large (or very small) density ratios, compute $\rho^{n+1}$ and $\mu^{n+1}$ from Eq. (38) (see Appendix A) and compute $c^{n+1}$ and $k^{n+1}$ by

$$
\left\{\begin{array}{l}
c^{n+1}=\frac{1}{2}\left(c_{1}+c_{2}\right)+\frac{1}{2}\left(c_{1}-c_{2}\right) \tilde{\phi}^{n+1} \\
k^{n+1}=\frac{1}{2}\left(k_{1}+k_{2}\right)+\frac{1}{2}\left(k_{1}-k_{2}\right) \tilde{\phi}^{n+1},
\end{array}\right.
$$

where $\tilde{\phi}^{n+1}$ is given by Eq. (37) (see Appendix A) .

4. Solve Eq. (33) (see Appendix A) for $P^{n+1}$.

5. Solve Eq. (35) (see Appendix A), together with the velocity Dirichlet condition (27b) (see Appendix A) on $\partial \Omega$, for $\mathbf{u}^{n+1}$.

6. Solve Eq. (16), together with the temperature Dirichlet condition (10b) on $\partial \Omega_{d}^{T}$, for $T^{n+1}$.

The new method has several notable characteristics. The computation for every flow variable involves only a constant and time-independent coefficient matrix for the linear algebraic system, which can be pre-computed during pre-processing. This effectively overcomes the performance bottleneck caused by variable coefficient matrices associated with variable thermal conductivity, variable specific heat, variable density, and variable viscosity. Only Helmholtz-type (including Poisson) equations with corresponding symmetric positive-definite matrices need to be solved within a time step using the algorithm. The computations for different flow variables are completely de-coupled. The method is suitable for large contrasts of the physical properties for the two fluids. The above features make the proposed method computationally efficient. 


\section{Numerical simulations}

In this section we use several numerical examples to test the new method developed for two-phase heat transfer problems in Section 2. The quantitative accuracy of the method is shown by the spatial and temporal convergence rate test. The physical accuracy of the method is established by simulating a laminar forced convection problem and a conjugate heat transfer problem and comparing results between simulation and analytic solution. Finally, a 3D simulation of transient cooling of a hot object immersed in water-air flow with a moving interface is presented. We take into consideration all crucial and realistic physical parameters such as surface tension, mixing energy density, specific heat and thermal conductivity. This example demonstrates that our method is capable of handling large density ratios up to three orders of magnitude and large viscosity ratios up to two orders of magnitude.

\subsection{Covergence tests}

We use an analytic solution to demonstrate the spatial and temporal convergence rates of the method developed in Section 2 for the two-phase heat transfer problem. To facilitate subsequent discussions, non-dimensional flow variables and physical parameters are used as shown in Appendix B.

We consider a flow domain, $-1 \leq x \leq 1,-1 \leq y \leq 1$ and $0 \leq z \leq 1$, as shown in Fig. 
2(a), and the following 3D analytic solution to the system of governing equations (1a)-(1d):

$$
\begin{cases}u=A \cos \left(a_{1} x\right) \sin \left(a_{2} y\right) \cos \left(a_{3} z\right) \sin \left(a_{4} t\right) & v=B \sin \left(a_{1} x\right) \cos \left(a_{2} y\right) \cos \left(a_{3} z\right) \sin \left(a_{4} t\right) \\ w=C \sin \left(a_{1} x\right) \sin \left(a_{2} y\right) \sin \left(a_{3} z\right) \sin \left(a_{4} t\right) & p=D \sin \left(a_{1} x\right) \sin \left(a_{2} y\right) \sin \left(a_{3} z\right) \cos \left(a_{4} t\right) \\ \phi=E \cos \left(b_{1} x\right) \cos \left(b_{2} y\right) \cos \left(b_{3} z\right) \sin \left(b_{4} t\right) & T=F \sin \left(d_{1} x\right) \sin \left(d_{2} y\right) \sin \left(d_{3} z\right) \sin \left(d_{4} t\right)\end{cases}
$$

where $(\mathrm{u}, \mathrm{v}, \mathrm{w})$ are the velocity components in $\mathrm{x}, \mathrm{y}$ and $\mathrm{z}$ directions, and $\mathrm{A}, \mathrm{B}, \mathrm{C}, \mathrm{D}, \mathrm{E}, \mathrm{F}, a_{i}$, $b_{i}, c_{i}(i=(1,4))$ are prescribed constants. Note that the expressions for $\mathrm{u}, \mathrm{v}$ and $\mathrm{w}$ satisfy Eq. (1b). In the governing equations, the body force $\mathbf{f}$ in (1a), the source terms $g$ in (1c) and $q_{s}$ in (1d) are chosen such that the analytic expressions in (19) satisfy the governing equations. Table 1 contains the specific values of all parameters, which result in Weber number $W_{e}=1.061 \times 10^{2}$, Cahn number $C_{n}=0.1$, Reynolds number $R_{e}=\frac{\rho_{1} U_{0} L}{\mu_{1}}=83.33$ and Peclet number $P_{e}=1.061 \times 10^{4}$. (see Appendix B for definitions).

Table 1: Specific values of parameters used for the contrived solution in convergence test.

\begin{tabular}{|l|l|l|l|}
\hline $\mathrm{A}=\mathrm{B}=\mathrm{C}=1.0$ & $\mathrm{D}=\mathrm{E}=\mathrm{F}=1.0$ & $a_{1}=a_{2}=\pi$ & $a_{3}=2.0 \pi$ \\
\hline$b_{1}=b_{2}=b_{3}=\pi$ & $d_{1}=d_{2}=d_{3}=\pi$ & $a_{4}=b_{4}=d_{4}=1.0$ & $h_{c}=0.1, T_{a}=1.0$ \\
\hline$\rho_{2} / \rho_{1}=3.0$ & $\mu_{2} / \mu_{1}=2.0$ & $k_{2} / k_{1}=3.0$ & $c_{2} / c_{1}=2.0$ \\
\hline
\end{tabular}

The set up for the boundary conditions is as follows: Dirichlet boundary condition (3) for the velocity is imposed on the domain boundaries, where the boundary velocity $\mathbf{w}$ is chosen according to the velocity analytic expression in (19). For the phase field functions $\phi_{1}$ and $\phi_{2}$, the contact-angle boundary conditions (4a) and (4b) with $\theta_{s}=60^{\circ}$ have been imposed on the domain boundaries, where the boundary source terms, $g_{a}$ in (4a) and $g_{b}$ in (4b), are chosen such that the analytic expression for the phase field function in (19) 
satisfies the boundary conditions (4a) and (4b). For the temperature, on the face $A B Q P$, DAPM, CFOR, DMJG, FILO, HILK we impose the heat flux boundary condition (7), where the heat flux $h_{n}$ is chosen such that the analytic expression in (19) satisfies (7). On the face $B C R Q$ we impose the convective heat transfer boundary condition (8), where the boundary source term $q_{r}$ is chosen such that the analytic expression in (19) satisfies (8). On the other faces we impose Dirichlet condition (6), where the boundary temperature $T_{d}$ is chosen according to the analytic expression for the temperature in (19).

We discretize the flow domain using four hexahedron spectral elements of equal size; see Fig. 2(a). We employ the algorithm developed in Section 2 for marching in time from $t=0$ to $\mathrm{t}=t_{f}\left(t_{f}\right.$ is a fixed time), and then compute the errors of the numerical solution at $t=t_{f}$ against the analytic solution given in (19).

In the first set of tests, we fix the time step size at $\Delta t=0.001$ and the final time instant at $t_{f}=0.1$ (i.e., 100 time steps). Then, we vary the element order systematically between 2 and 12. In Fig. 2(b) we show the $L^{2}$ errors of the computed results for the velocity, pressure, phase field function and temperature $t=t_{f}$ as a function of the element order. We observe that as the element order increases (but below order 12), the numerical errors of the solution decrease exponentially. As the element order increases to 12 and beyond, the error curves level off because the total error has been saturated by the truncation error in time. The saturation error $10^{-6}$ here is due to temporal accuracy, which is second-order for $\Delta t=10^{-3}$ since we use a second-order backward time scheme for time discretization. These results demonstrate the spatial exponential convergence rate of our method.

In the second set of tests, we fix the element order at 10 and final time at $t_{f}=1.0$. Then we vary the time step size systematically between $\Delta t=0.00625$ and $\Delta t=0.05$. Fig. 2 (c) shows 
$L^{2}$ errors of the numerical solutions for all flow variables as a function of time step size in logarithmic scales. It is evident that our method achieves second-order convergence rate in time. 


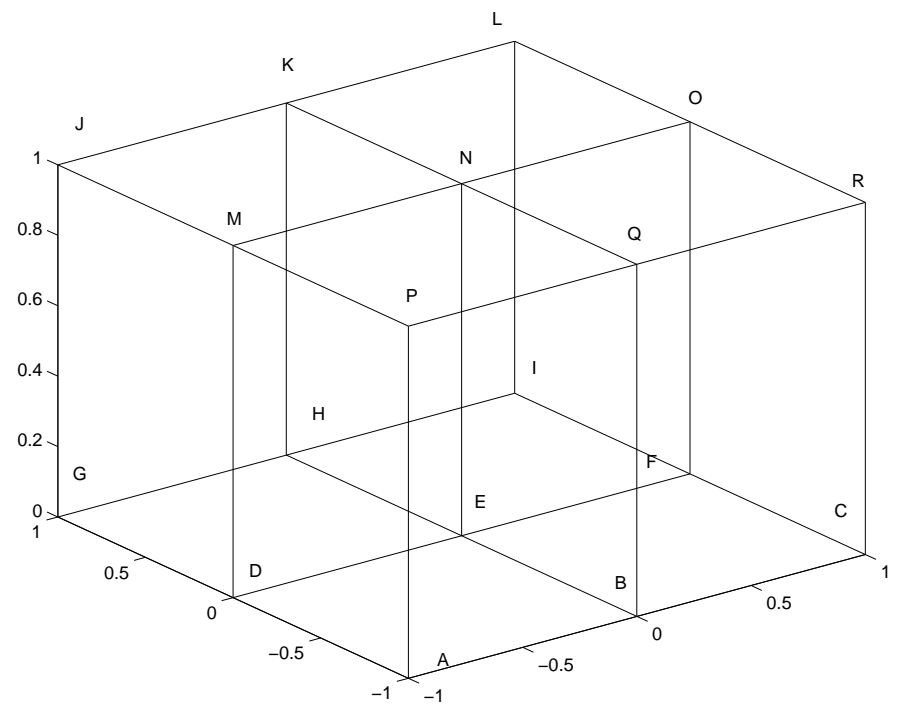

(a)

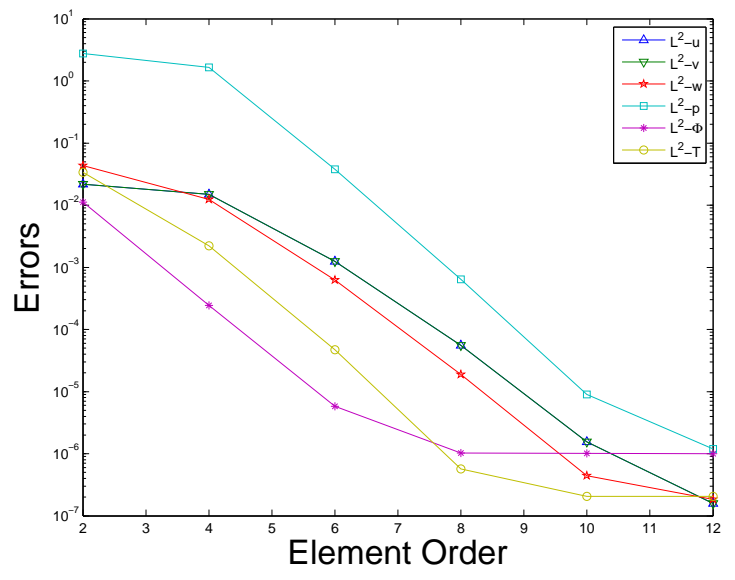

(b)

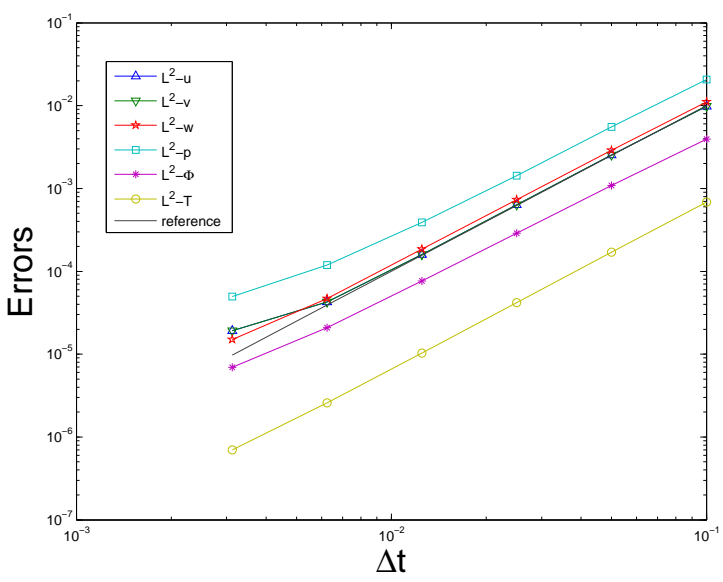

(c)

Figure 2: Convergence rates: (a) Mesh; (b) Numerical errors versus element order (fixed $\Delta t=0.001) ;(c)$ Numerical errors versus $\Delta t$ (fixed element order 10). The saturation of the convergence rate in (b) is due to temporal errors. 


\subsection{Laminar forced convection}

In this subsection, we use a laminar forced convection problem to test our algorithm developed in Section 2 for two-phase temperature equation (1d). We assume a core-annular configuration for a flow with fully developed velocity profile but developing temperature profile. Convection is due to prescribed pipe wall temperature $T=0$ and fluid temperature $T=1$ at the inlet $z=0$. In this section, we consider flow in a pipe geometry. Detailed initial and boundary conditions for velocity, phase field and temperature are given in Appendix C. We have also simulated single-phase laminar forced convection in a rectangular duct for different temperature boundary conditions and compared the Nusselt numbers with empirical values given in [24]; these results are included in Appendix D.

More specifically, we consider a circular pipe with radius $0.5 \mathrm{~L}$ and length $10 \mathrm{~L}$ ( $\mathrm{L}$ is the characteristic length and will be specified later), which occupies the domain $\Omega=\{(r, z)$ :

$\left.0 \leq r \leq \frac{L}{2}, 0 \leq z \leq 10 L\right\}$. Boundary source terms for phase field and for temperature $\left(g_{a}\right.$ in (4a), $g_{b}$ in (4b) and $\left.q_{r}(8)\right)$ are all set to be zero and the effect of gravity is neglected. Interpolation with polynomial order 3 has been used for all the elements. A time step size $\Delta t=10^{-3}$ has been used in the simulation.

The characteristic length $\mathrm{L}$ is chosen to be $1 \mathrm{~m}$ and characteristic velocity $U_{0}=1.0 \mathrm{~m} / \mathrm{s}$, which is also the average velocity on cross sections.

To simulate this problem, we discretize the domain with 9150 hexahedron elements (305 elements in $\mathrm{x}-\mathrm{y}$ plane and 30 elements in $\mathrm{z}$ ). The mesh is denser near the interface area at radius $r=0.4$ and sparser otherwise (relatively at 10 times scale). The presence of immiscible fluids in our problem implies sharp interface (i.e., zero interface thickness). However, the phase-field model we adopted for the two-phase Navier-Stokes flows assumes a thin smooth 
transition layer (i.e., nonzero interface thickness) connecting the two immiscible fluids [21]. In [25], it is shown that a necessary condition for solutions from phase-field modeling to approach those of the original sharp-interface Navier-Stokes equations is to reduce the mesh size at a faster rate than the interface thickness. Therefore, we use mesh size at scale $10^{-3}$ near the interface area to suppress the effects from the grid.

In the first subsection, we derive an analytic solution for the temperature field based on the eigen-problem given in [26]. Then, we simulate a single-phase flow forced convection. The second subsection is devoted to simulating a two-phase flow forced convection. For both single-phase and two-phase cases, we will present the results by comparing the solutions of temperature fields between the simulation and the analytic solution.

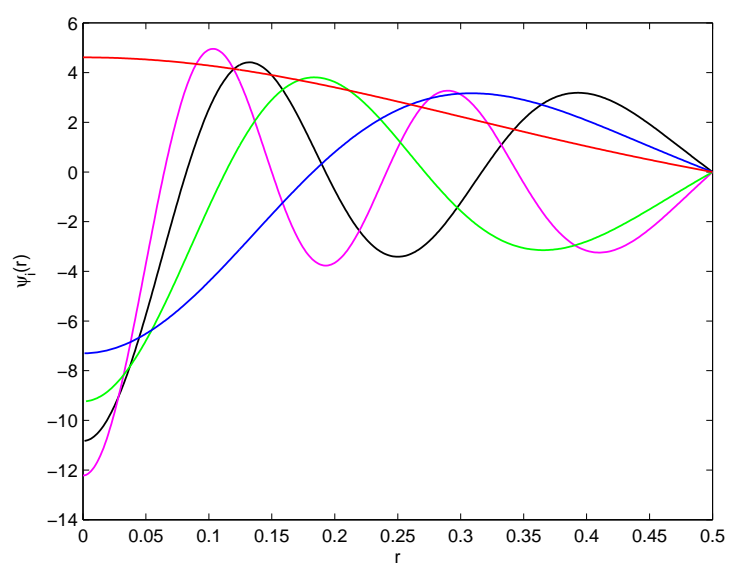

(a)

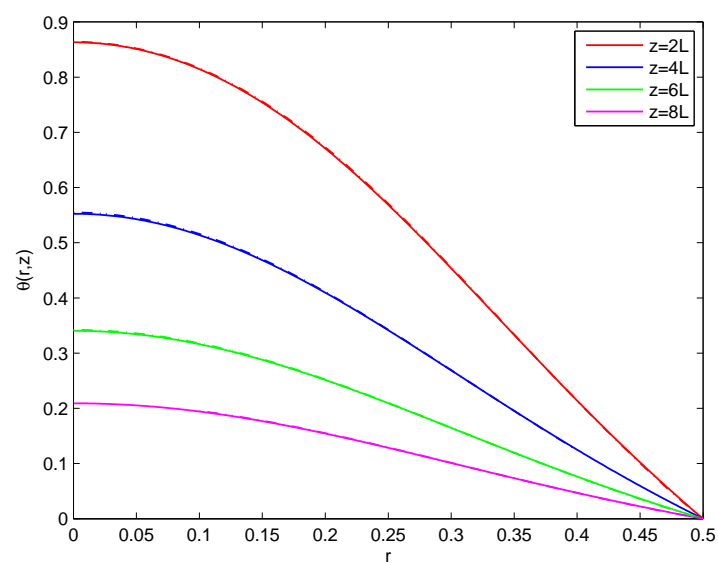

(b)

Figure 3: (a) The first five temperature eigen-modes $\left(\psi_{i}(r)\right)$ for single-phase flow at $R e=60$.

(b) Comparison of temperature profiles at different cross sections with exact solution for single phase flow at $P_{r}=1, R e=60$. Solid line: exact solution; dashed line: present computation. 


\subsubsection{Single-phase laminar forced convection}

We consider a single-phase flow in the circular pipe with density $\rho=1.0$, dynamic viscosity $\mu=0.017$, thermal conductivity $k=0.017$ and specific heat $c=1.0$. The resulted Reynolds number $R_{e}$ is 60.0 and Prandtl number $P_{r}$ is 1.0 .

We obtain the analytic solution for temperature by solving the following eigen-value problem given in [26] :

$$
\frac{d}{d r}\left(\alpha_{m} \frac{d \psi_{i}}{d r}\right)+\lambda_{i}^{2} r w(r) \psi_{i}(r)=0
$$

where $\psi_{i}(r)$ (corresponding to the eigenvalues $\lambda_{i}$ ) are the eigen-functions obtained from equation (20).

Fig. 3(a) shows the first five temperature eigen-modes for a single-phase flow. We construct the analytic solution for temperature $\theta(r, z)$ in terms of these eigen-modes as follows:

$$
\theta(r, z)=\sum_{i=1}^{N} a_{i} \psi_{i}(r) \exp \left(-\lambda_{i}^{2} z\right)
$$

where $\theta(r, z)$ is the solution for the temperature field. Fig. 3(b) shows the comparison of temperature profiles at different cross sections along the pipe between our simulation and the analytic solution for single-phase flow. We see that temperature profiles from our simulation virtually overlap with those from the analytic solutions. 


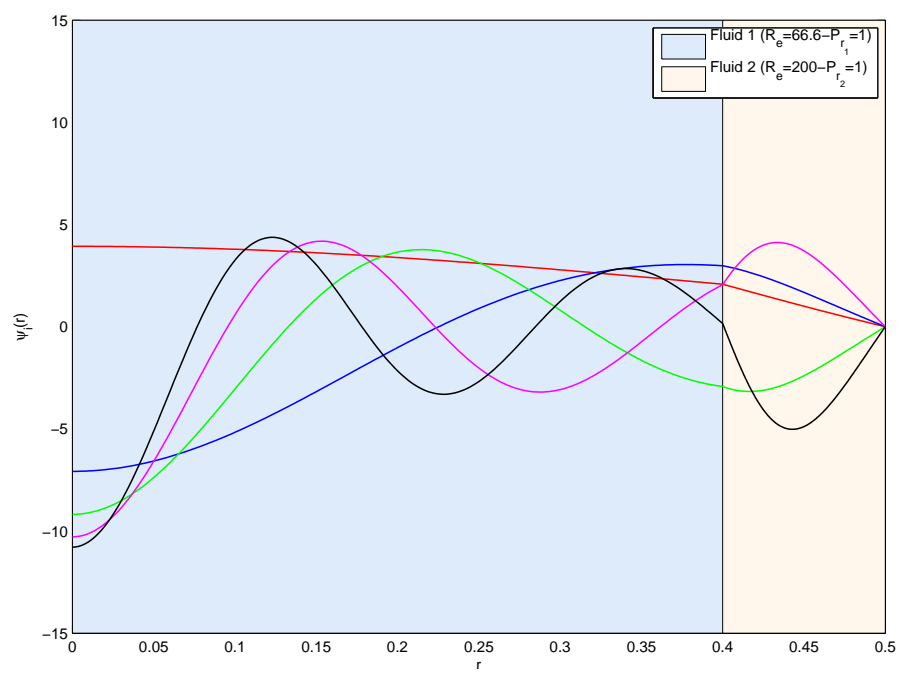

Figure 4: The first five eigen-modes $\left(\psi_{i}(r)\right)$ for temperature.

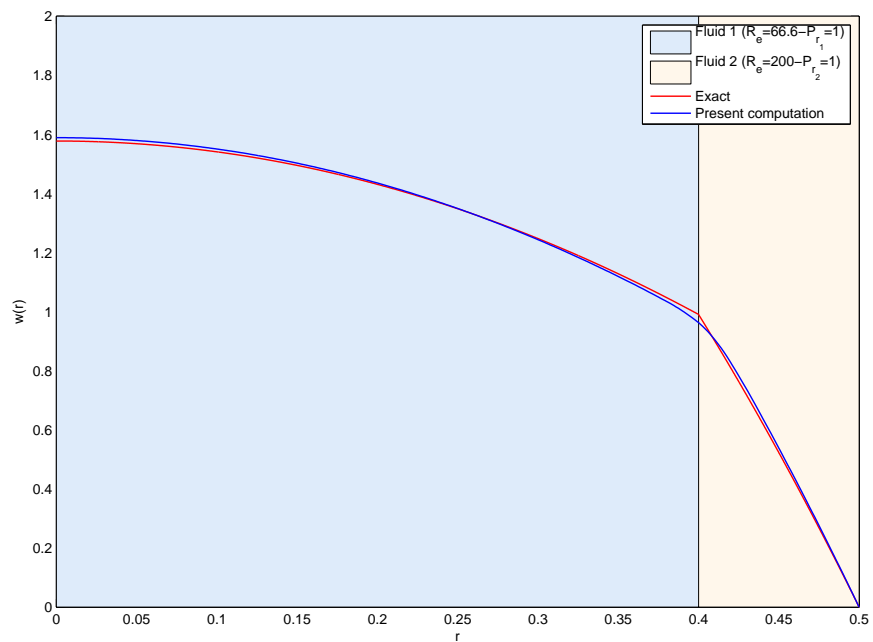

Figure 5: Fully developed axial velocity along the pipe. 


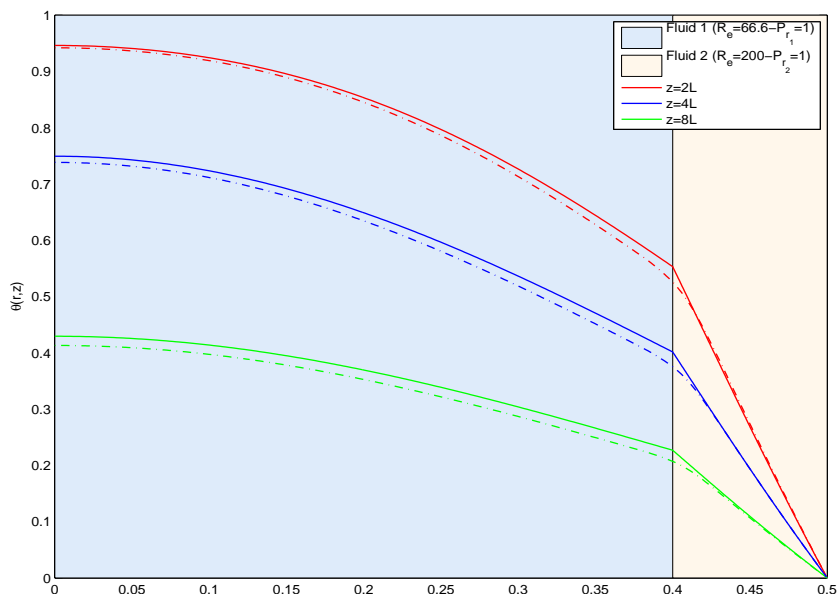

Figure 6: Comparison of temperature profiles at different sections with exact solution for two-phase flow with the inside fluid at $P_{r}=1, R_{e}=66.66$ and the outside fluid at $P_{r}=$ $1, R_{e}=200$. Interface thickness $\eta=0.01$. Solid line: exact solution; dashed line: present computation.

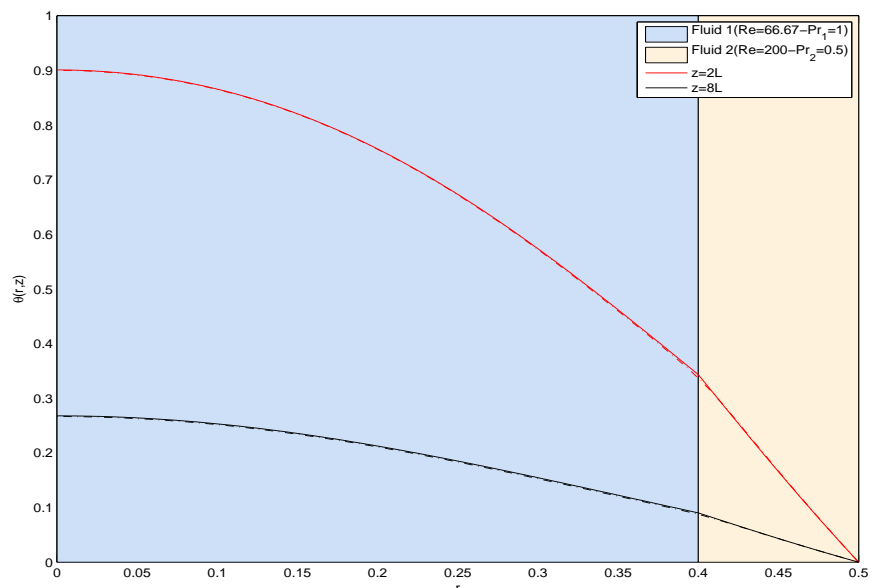

Figure 7: Comparison of temperature profiles at different sections with exact solution for two-phase flow with the inside fluid at $P_{r}=1, R_{e}=66.66$ and the outside fluid at $P_{r}=$ 0.5, $R_{e}=200$. Interface thickness $\eta=0.01$. Solid line: exact solution; dashed line: present computation. 


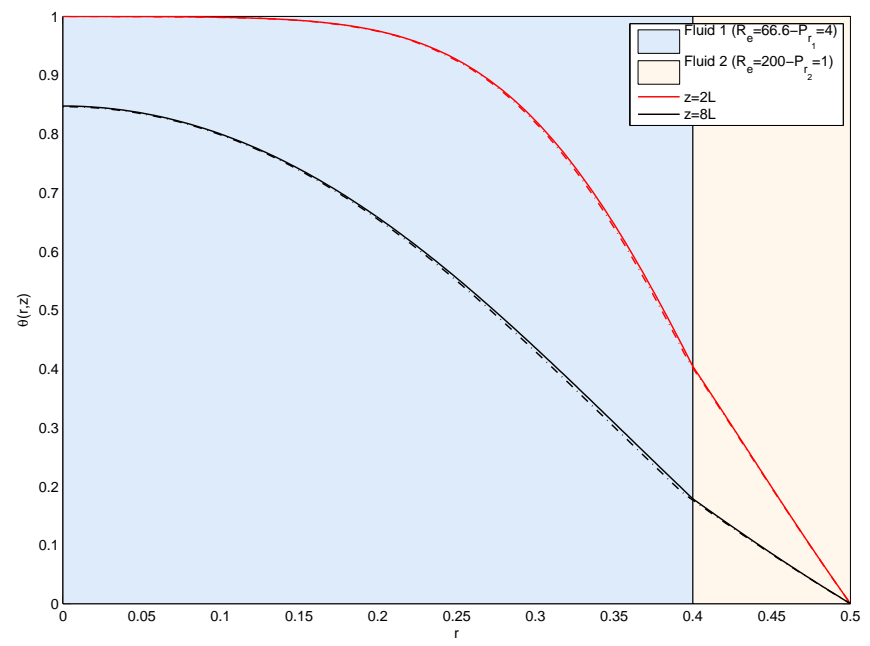

Figure 8: Comparison of temperature profiles at different sections with exact solution for two-phase flow with the inside fluid at $P_{r}=4, R_{e}=66.66$ and the outside fluid at $P_{r}=$ $1, R_{e}=200$. Interface thickness $\eta=0.01$. Solid line: exact solution; dashed line: present computation.

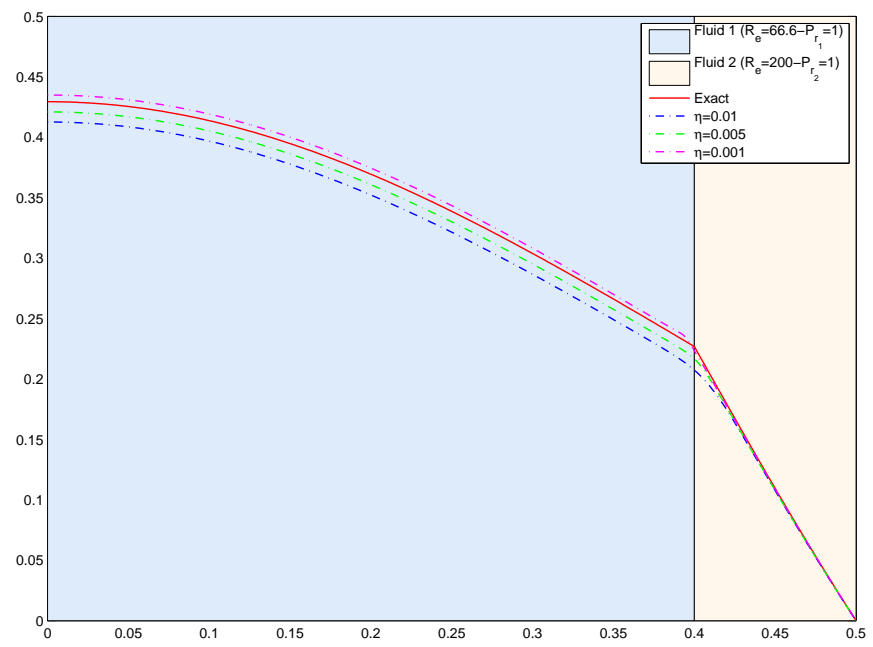

Figure 9: Effect of interface thickness. Comparison of temperature profiles at cross section $z=8 L$ with exact solution for two-phase flow. Solid line: exact solution; dashed line: present computation. 


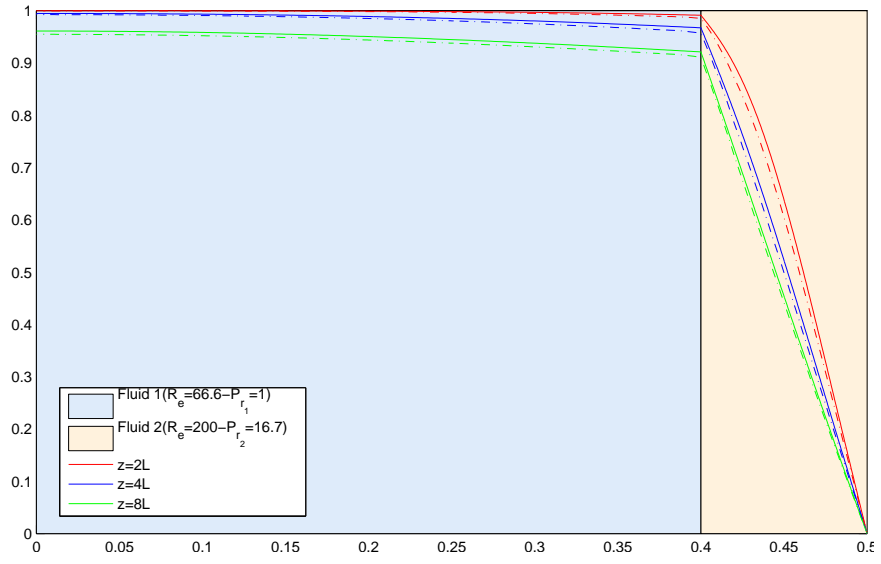

Figure 10: Thermal diffusivity ratio 50 using a uniform mesh. Comparison of temperature profiles at different cross sections with exact solution for two-phase flow with the inside fluid at $P_{r}=1, R_{e}=66.66$ and the outside fluid at $P_{r}=16.7, R_{e}=200$. Interface thickness $\eta=0.001$. Solid line: exact solution; dashed line: present computation.

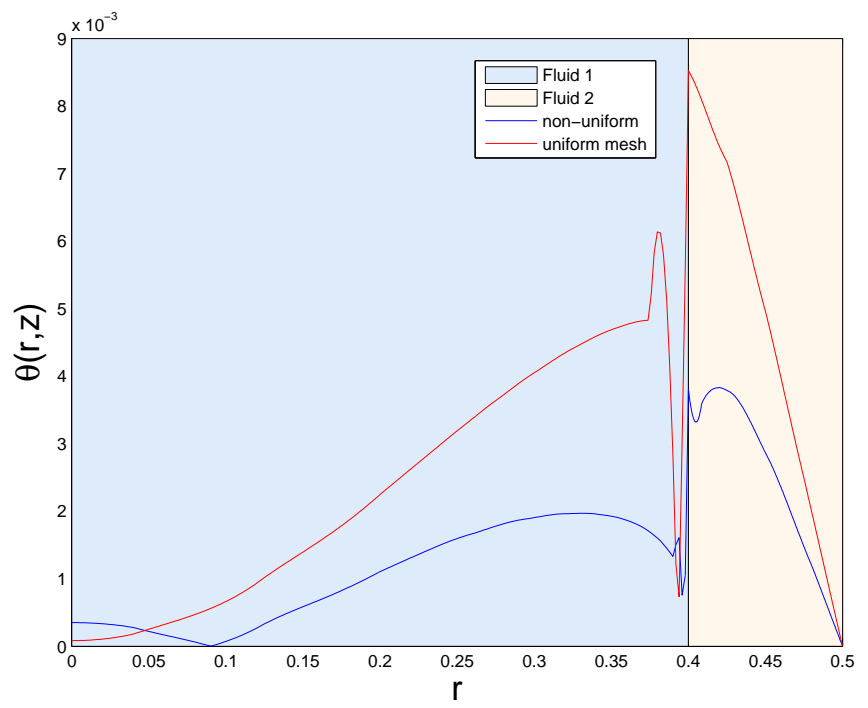

Figure 11: Effect of the mesh size near the location of the interface. Absolute difference of the results between computation and exact solution from uniform mesh and non-uniform mesh at cross section $\mathrm{z}=2 \mathrm{~L}$ for two-phase flow with the inside fluid at $P_{r}=1, R_{e}=66.66$ and the outside fluid at $P_{r}=1, R_{e}=200$. Interface thickness $\eta=0.001$. 


\subsubsection{Two-phase laminar forced convection}

Here we test our method by simulating forced convection for two co-flowing immiscible fluids in a pipe. The goal is to demonstrate the physical accuracy of our method in dealing with the two-phase heat transfer problem.

We use the same core-annular configuration as in the last subsection. We assume two fluids in the pipe with one fluid surrounded by the other. We refer to the fluid inside as inside fluid or the first fluid and the fluid that surrounds the inside fluid to be outside fluid or the second fluid. The analytic solution for the temperature field is obtained in the same manner as in the last subsection. Convection cools down the fluid inside along the axial direction. Phase change does not take place and the effect of gravity is neglected. An element of polynomial order 3 has been used for all the elements. A time step size $\Delta t=10^{-3}$ has been used in the simulation.

The initial phase field profile is given by the following hyperbolic tangent function:

$$
\phi(x, y, z ; t=0)=-\tanh \left(\left(\sqrt{\left(x^{2}+y^{2}\right)}-r_{1}\right) / \sqrt{2} \eta\right)
$$

where $r_{1}=0.4$ is the radius position for the initial position of the interface and $\eta$ is the interface thickness between the two fluids. We note here that the initial phase field profile function in Eq. (22) varies continuously over the transition layer and is mostly uniform in the bulk phases, consistent with the assumption of the phase-field modeling in our approach. Consequently, solutions for all flow variables (including temperature) in our simulation exhibit the same behavior. However, the sharp interface (i.e., zero interface thickness) for immiscible fluids in reality leads to a discontinuity in the analytic solutions at which physical parameters change abruptly from one fluid to another. This can be seen in Fig. 4, where 
each of the first five eigen-modes for temperature has a discontinuity at the interface $r=0.4$. Correspondingly, there is a discontinuity in the analytic solution for temperature based on these eigen-modes at the same place $r=0.4$. Therefore, we can predict that there will be an error in our comparison which is due to the smooth phase-field modeling in our method. In [25] it is shown that for phase field functions the error from the nonzero interface thickness assumption can be reduced if the interface thickness goes to zero under certain conditions. In our method, the temperature field is calculated based on the resolved phase field functions. Therefore, for temperature, the error between the simulation and the analytic solution shall decrease if the error for phase field function decreases. In other words, we expect the interface thickness to play the same role for the solutions of temperature field as it does for the phase field function. We will investigate the effect of the interface thickness at the end of this subsection.

Table 2 shows all the parameters we use. These parameters result in density ratio $\rho_{2} / \rho_{1}=1$, dynamic viscosity ratio $\mu_{2} / \mu_{1}=3.0$, specific heat ratio $c_{2} / c_{1}=1.0$ and thermal conductivity ratio $k_{2} / k_{1}=3.0$. Also, we have surface tension $\sigma=0.001$ and interface thickness $\eta=0.01$. Here we would like to point out that we use surface tension and interface mobility as small as $10^{-3}$ for this problem.

Since we concentrate on the analysis of a laminar forced convection, we first check that the velocity field and phase-field from our simulation are in agreement with the analytic solution. Fig. 5 shows the fully developed velocity profile of the two fluids from the simulation and the analytic solution given in [26]. We see that the results for the velocity profiles from the simulation achieve good agreement with those from the analytic solution except of a slight difference near the interface. The initial phase-field profile in our simulation is the same 
Table 2: Hydrodynamic and thermal properties of the two-phase flow in a pipe.

\begin{tabular}{|l|l|l|l|l|l|l|}
\hline & $\begin{array}{l}\text { Density } \\
\left(\mathrm{kg} / \mathrm{m}^{3}\right)\end{array}$ & Dynamic Viscos- & Thermal Con- $(\mathrm{kg} /(\mathrm{m} \cdot \mathrm{s}))$ & Specific heat & Reynolds & Prandtl \\
& ductivity & $(\mathrm{J} / \mathrm{kg} \cdot \mathrm{m} \cdot \mathrm{K}))$ & & \\
\hline Inside & 1.0 & 0.015 & 0.015 & 1.0 & 66.67 & 1.0 \\
\hline Outside & 1.0 & 0.005 & 0.005 & 1.0 & 200 & 1.0 \\
\hline
\end{tabular}

as the co-current flow profile but we have a small transition layer (i.e., nonzero interface thickness) between the two fluids. In our simulation, this phase-field profile persists over time.

Now let us look at the temperature. We first show the results from the case with matched Prandtl numbers for both fluids at a fixed interface thickness. Fig. 6 compares the temperature field obtained from the simulation and the analytic solution across different cross sections along the pipe. In two bulk phases, results from both the simulation and the analytic solution exhibit different decaying rates. The decaying rate is larger for the outside fluid (i.e., the second fluid) than for the inside fluid (i.e., the first fluid). This comes from the fact that the diffusivity coefficient of the outside fluid is different from that of the inside fluid.

Also, in the domain of the outside fluid, we see a good match between the results from the simulation and the analytic solution. However, when crossing the interface area, results from the simulation deviate slightly from the analytic solution. This difference is carried along in the entire domain of the inside fluid. As discussed above, this might be due to the 
nonzero interface thickness assumption in the phase-field model.

Let us next consider different Prandtl numbers for the two fluids. Fig. 7 shows the results with the inside fluid at Prandtl number 1 and the outside fluid at Prandtl number 0.5. Fig. 8 shows the results with the inside fluid at Prandtl number 4 and the outside fluid at Prandtl number 1. All parameters employed are the same as in Table 2 except that thermal conductivities are changed to get different Prandtl numbers. Results from the simulation and the analytic solution are in good agreement with each other. Note that the difference between the results from the simulation and the analytic solution is smaller in these two cases than the previous case with matched Prandtl number for both fluids. This is because the difference of the thermal diffusivity of the two fluids is smaller in the latter two cases than before. This is also shown by the smaller difference of the slope of the lines in two bulk phases in these two cases than before.

Next, let us look closer at the effect of interface thickness in our simulation. All parameters are the same as in Table 2 except changes for $\eta$. Fig. 9 shows that interface thickness $\eta=0.005$ brings the results from simulation closer to those from the analytic solution than interface thickness $\eta=0.01$. If we further decrease the interface thickness to $\eta=0.001$, the absolute difference between the simulation and the analytic solution shrinks even more. This confirms our previous prediction that the temperature solution from the simulation can approach that of the original sharp-interface Navier-Stokes equations as the interface thickness goes to zero.

Next, we perform a test for the thermal diffusivity ratio 50 for the two fluids, which is much larger than the thermal diffusivity ratio 3 we used for the above tests. The goal is to examine the accuracy of our model in handling large thermal diffusivity ratio problems. 
All parameters employed are the same as in Table 2 except that the thermal conductivity of the outside fluid is decreased to 0.0003. From Fig. 10, we see a good match between our calculation and the exact solution.

Finally, we compare the results between the non-uniform mesh (as used in the above calculations) with an uniform mesh. By non-uniform mesh we refer to smaller mesh size near the interface and by uniform mesh we refer to approximately same mesh size near the interface as other places. The mesh size near the interface of the non-uniform case is about $1 / 3$ of the uniform case. The goal of this test is to investigate the effects of the mesh size near the interface. All parameters employed are the same as in Table 2. From Fig. 11, we see that the absolute difference of the temperature profile between computation and exact solution from the non-uniform mesh is smaller than the uniform mesh but the magnitude of the difference is only $10^{-3}$. Therefore, even if for real flow problems in which we can no longer use locally refined mesh, we still can achieve good simulation results provided sufficiently small mesh size and appropriate interface thickness are used.

The simulation results of this section demonstrate that our algorithm can be an effective and accurate method for simulating two-phase convective heat transfer problem. It allows the accurate calculation of two-phase convective heat transfer with a thin interface thickness as small as $10^{-3}$.

\subsection{Conjugate heat transfer}

Next we consider simulating a conjugate heat transfer problem. The goal is to further validate the accuracy and robustness of our method.

Fig. 12(a) shows the $\mathrm{y}-\mathrm{z}$ plane of the computational domain, which is $-0.1 \leq x \leq 0.1$, 
$-0.5 \leq y \leq 0.5$, and $0 \leq z \leq 3$. Fluid initially fills the domain from $y=-0.5$ up to $y=0.0$. A solid object occupies the domain from $y=0.0$ to $y=0.5$. We assume that the flow is fully developed by adding a driving force in the $\mathrm{z}$ direction.

For velocity, the bottom and top sides of the domain $(y=-0.5$ and $y=0.5)$ are solid walls, while the other sides $(x= \pm 0.1, z=0$ and $z=3)$ are all periodic boundaries. For temperature, we assume the bottom wall has temperature $T=1$ and the top wall has temperature $T=0$, while all other sides are periodic boundaries. Initially we impose $T=0$ for the entire domain. The setup of this problem models a heat transfer process that a solid object cools down by the flow of the fluid below. As for the phase field, we assume the fluid to be the first phase and the solid object to be the second phase. There is no explicitly imposed boundary condition at the interface between solid and fluid.

The values used for the physical parameters for this problem are shown in table 3.

Table 3: Non-dimensional hydrodynamic and thermal parameters of conjugate heat transfer problem.

\begin{tabular}{|l|l|l|l|l|}
\hline & Density & Dynamic Viscosity & Thermal Conductivity & Specific heat \\
\hline Solid & 1.0 & & 0.005 & 1.0 \\
\hline Fluid & 1.0 & 0.01 & 0.015 & 1.0 \\
\hline
\end{tabular}

To simulate this problem, we discretize the computational domain into 400 hex elements with 2 elements in $\mathrm{x}$ direction, 20 elements in y direction and 10 elements in z direction. The expansion order is 3 for all the elements. The non-dimensional time step size is $\Delta t=0.001$. 
We have performed the simulation for total computational time $t=80$ by employing the method developed in Section 2.

First of all, we look at the velocity profile in our simulation to make sure it is consistent with our assumption. The analytic solution of velocity for our problem is $U_{x}=0, U_{y}=0$, and $U_{z}=a^{2} /(2.0 \mu)\left(-(y / a)^{2}-y / a\right)$, where $a=0.5$ is the length of the cross section of the fluid domain. As shown in Fig. 12(b), the velocity profile at time $t=80.1$ from our simulation overlaps with the analytic solution.

Then we compare our results of the temperature distribution with the analytic solution, which we construct following the method in [27, 28]. Fig. 12(c) shows the temperature profile at the $\mathrm{x}-\mathrm{y}$ plane cut at $z=1.5$ at different times from our simulation and analytic solution. Results from the present computation and analytic solution overlap with each other. This shows the ability of our algorithm to handle problems involving both conduction in solid and convection in fluid and their interaction.

Finally, we also perform a test for a tri-layer heat transfer problem. The geometry is the same as above. The physical parameters used are the same except for the solid part we have thermal conductivity 0.01 from $y=0$ to $y=0.25$ and 0.005 from $y=0.25$ to $y=0.5$. Fig. $12(\mathrm{~d})$ shows the results from our simulation agree well with those from analytic solution. This demonstrates the ability of our method in handling variable thermal conductivities in conjugate heat transfer problems.

\subsection{Transient cooling of a hot object in a 3D cavity}

Here we demonstrate the capability of our method to simulate a realistic 3D heat transfer system containing cold water and hot air with the moving air-water interface. In particular, 
we have a large density ratio of 829 and a large viscosity ratio of 56 between the two fluids. The computational domain is a three-dimensional cavity given by $\Omega=\{(x, y, z): 0 \leq x \leq$ $2,0 \leq y \leq 3,-0.5 \leq z \leq 0.5\}$. Inside the cavity, a hot object is placed. The schematic of the problem at the $\mathrm{x}-\mathrm{y}$ plane with $\mathrm{z}=0$ is shown in Fig. 13.

Initially water is filled to $30 \%$ of the box from the bottom and the rest of the domain is occupied up by air. We assume that initially the water has a lower temperature of $T_{W}=20^{\circ} \mathrm{C}$ and the air has the higher temperature of $T_{A}=25^{\circ} \mathrm{C}$. A water jet is flushed from the inlet at the bottom left of the domain (see Fig. 13), raises the water surface up and pushes the air out from the outlet at the top left of the domain. The hot object is extended in the z-direction in the range of $-0.5 \leq z \leq 0.5$. The hot object in the middle of the cavity heats up the water and air, by assuming a constant heat flux on its side walls. In our mathematical model, the buoyancy effect between water and air due to their density difference has also been considered.

The initial phase-field profile is given by the following hyperbolic tangent function:

$$
\phi=\tanh \left(\left(y-Y_{0}\right) /(\sqrt{2} \eta)\right)
$$

where $Y_{0}$ is the initial position of water surface and $\eta$ is the initial interface thickness between water and air. For the phase-field function, the contact-angle boundary condition of $\theta_{s}=90^{0}$ is imposed on all the domain boundaries, where the boundary source terms $g_{a}$ and $g_{b}$ are set to be zero. Initially, the temperature profile is set up as follows:

$$
T=0.5\left(T_{A}+T_{W}\right)+0.5\left(T_{A}-T_{W}\right) \tanh \left(\left(y-Y_{0}\right) /(\sqrt{2} \eta)\right) .
$$

More details of the position and center of the inlet and outlet, the initial and boundary conditions for the velocity and temperature fields are presented in Appendix E. The 
physical parameters are listed in table 4. In addition, we use surface tension $\sigma=37.79$,

Table 4: Non-dimensional hydrodynamic and thermal parameters of the air-water system in the 3D cavity.

\begin{tabular}{|l|l|l|l|l|}
\hline & Density & Dynamic Viscosity & Thermal Conductivity & Specific heat \\
\hline Air & 1.0 & 0.06 & $8.5 \times 10^{-2}$ & 1.0 \\
\hline Water & 829.01 & 3.47 & 2.04 & 4.13 \\
\hline
\end{tabular}

and interface thickness $\eta=0.02$. These parameters result in density ratio $\rho_{2} / \rho_{1}=829.01$, dynamic viscosity ratio $\mu_{2} / \mu_{1}=56.29$, specific heat ratio $c_{2} / c_{1}=4.13$ and thermal conductivity ratio $k_{2} / k_{1}=24.00$. In addition to those non-dimensionalized parameters, we use the Weber number $W_{e}=2.65 \times 10^{-2}$, Peclet number $P_{e}=4.24 \times 10^{2}$, Cahn number $C_{n}=0.02$, Reynolds number $R_{e}=\frac{\rho_{1} U_{0} L}{\mu_{1}}=16.24$ and Froude number $F_{r}=0.32$ (see Appendix A for definition).

For spatial resolution, we use 5400 hexahedral elements with spectral polynomial order $P=3$. A time step size $\Delta t=10^{-3}$ has been used in the simulation.

In Fig. 14, nine snapshots of phase-field distribution at different time instants are shown. These snapshots are taken at the mid span-wise plane $(\mathrm{z}=0)$. Initially the water and air are stagnant, with water occupying the bottom of the cavity and the air occupying the remaining of the domain (see Fig. 14(a)). As the inlet water jet turns on, the water level begins to rise from both sides of the hot object. At the bottom of the hot object, a low-velocity region forms due to the presence of boundary layer near the wall and the flow blockage caused by 
the wall. As a result, a thin pocket of air is trapped underneath the hot object (see Fig. 14(b)). Due to the large density ratio between air and water, the air pocket, enclosed by the wall and the surrounding water, is highly unstable. The velocity distribution around the hot object is asymmetric due to the asymmetric location of the inlet boundary. This asymmetry in velocity provides a pathway for the trapped air to escape from the left side of the hot object (see Fig. 14(b) and Fig. 14(c)). The large buoyancy force applied from water to air creates a large-velocity region near the left surface of the hot object, which can be seen in Fig. 14(d)-Fig. 14(f). Once the water level reaches above the hot object, an overflow of the water on hot object can be observed, as shown in Fig. 14(g)-Fig. 14(i).

In Fig. 15, temperature distributions, at the same time instants as those of Fig. 14, are shown. At initial condition, the water is at temperature $T_{W}=20^{\circ} \mathrm{C}$ and air at $T_{A}=25^{\circ} \mathrm{C}$, as shown in Fig. 15(a). As time advances, the temperature of the air rises faster than the water due to the lower heat capacity of air. As a result, the temperature of the air pocket underneath the hot object is slightly above the surrounding water (see (Fig. 15(b) and Fig. 15(c))). The flow on the top surface of the hot object is nearly stagnant, which results in the highest temperature increase in a nearly diffusing manner at the early stages of the simulation. As the water rises through the cavity, the high-temperature air above the hot object gets convected away and is replaced by the "rushing" low-temperature water that overflows the hot object (see Fig. 15(d)-Fig.15(f)). As the air overflows the hot object, two counter-rotating vortices begin to form on two sides of the cavity, as it can be seen in Fig. 15(g)-Fig. 15(i).

Fig. 16 shows velocity snapshots corresponding to the same instants as those in the last three plots in Fig. 15. The emergence of the two counter-rotating vortices from either side 
of the cavity can be clearly seen. The overflow of two opposing streams of water on the top surface of the hot object creates a stagnation point in this region. The opposing streams of water induce a shear flow in the air above the interface as it can be seen in Fig. 16.

In Fig. 17, a horizontal cross section of the computational domain at $y=2.05$ is shown just above the hot object. The snapshots show the phase-field and the $(u-w)$ velocity vectors at $t=10.1$. At this time instant, the bulk of the air pocket has already left the horizontal plane $y=2.05$. However, due to the upward movement of the air pocket, strong shear is induced on the nearby water. Moreover, a void is created by the leaving air pocket. Both of these effects cause a trailing upward movement of the water. The flushing of the water can be clearly seen in Fig. 17(a). The penetration of the trailing water stream into the air is not possible since the water is negatively buoyant in air. Instead, water spreads horizontally over the hot object. In the two-dimensional view $(x-z)$, the vertical water stream behaves as a mass source, creating a positive two-dimensional divergence of the velocity field $\left(\nabla_{x z} \cdot \mathbf{u}=\partial u / \partial x+\partial w / \partial z>0\right)$ at the point of injection. 


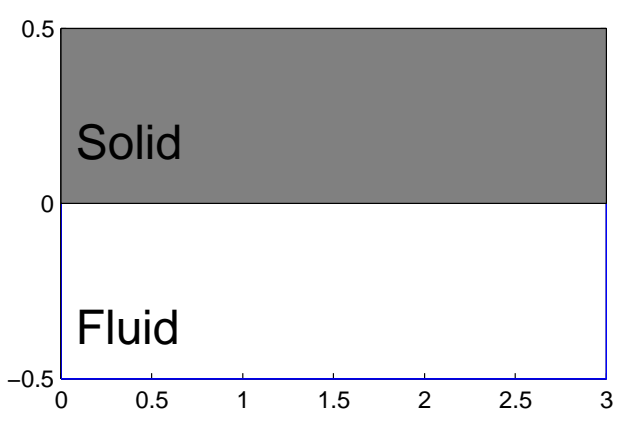

(a)

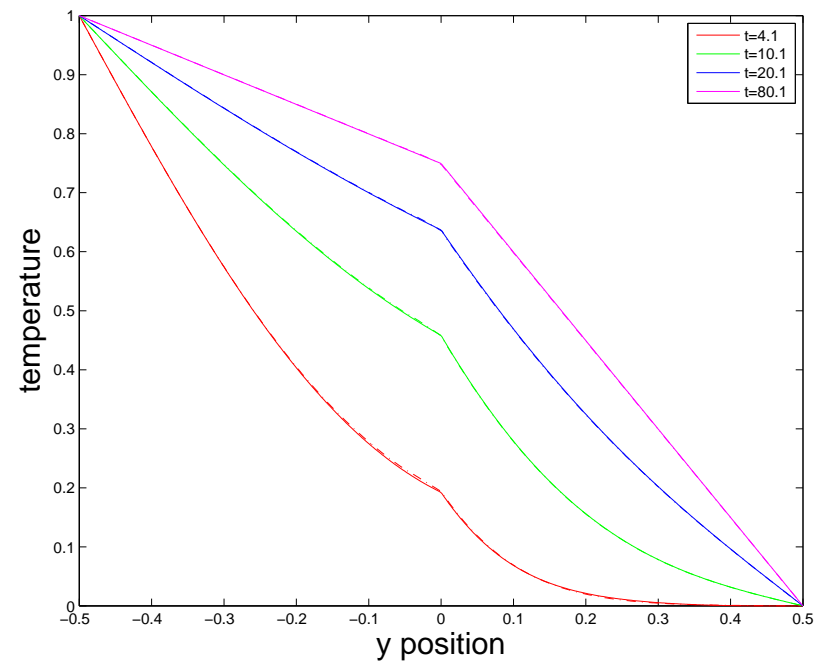

(c)

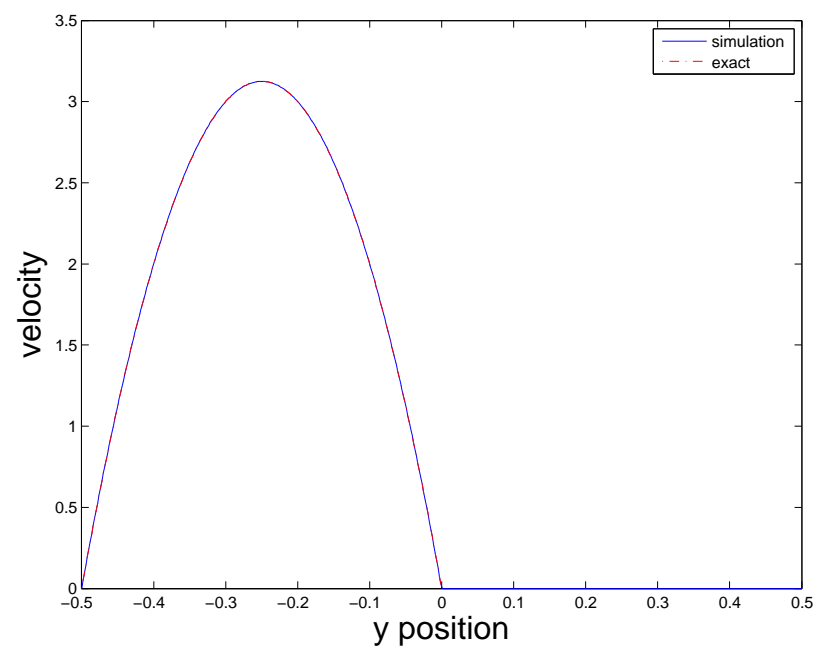

(b)

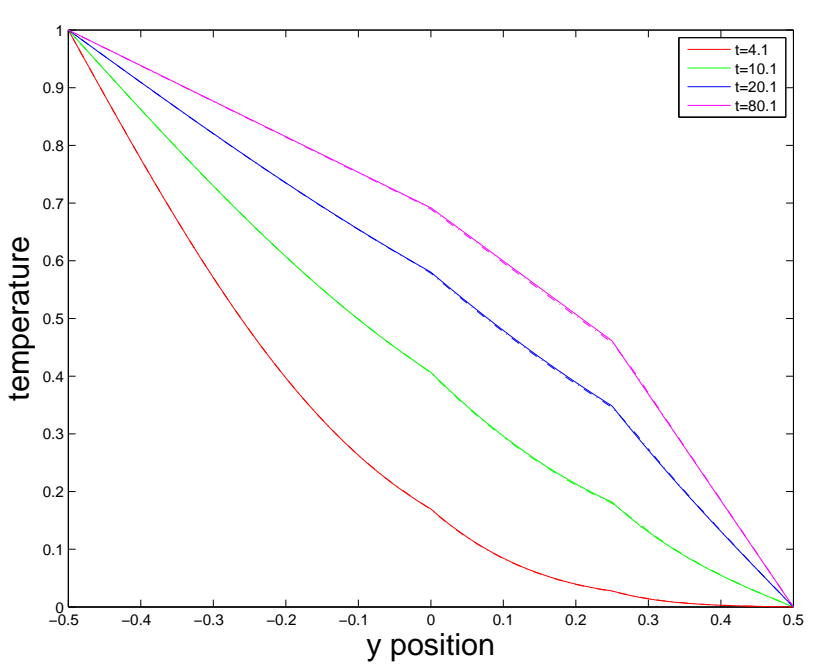

(d)

Figure 12: Simulation of a conjugate heat transfer problem. (a) Mesh; (b) velocity profile at $\mathrm{x}-\mathrm{y}$ plane cut at $z=1.5$ at time $t=80.1$; (c) temperature profile at $\mathrm{x}-\mathrm{y}$ plane cut at $z=1.5$ at different times. (d) temperature profile at $x-y$ plane cut at $z=1.5$ at different times for the tri-layer heat transfer problem. Solid line: exact solution; dashed line: present computation. Results from present computation and analytic solution are graphically indistinguishable in (b), (c) and (d). 


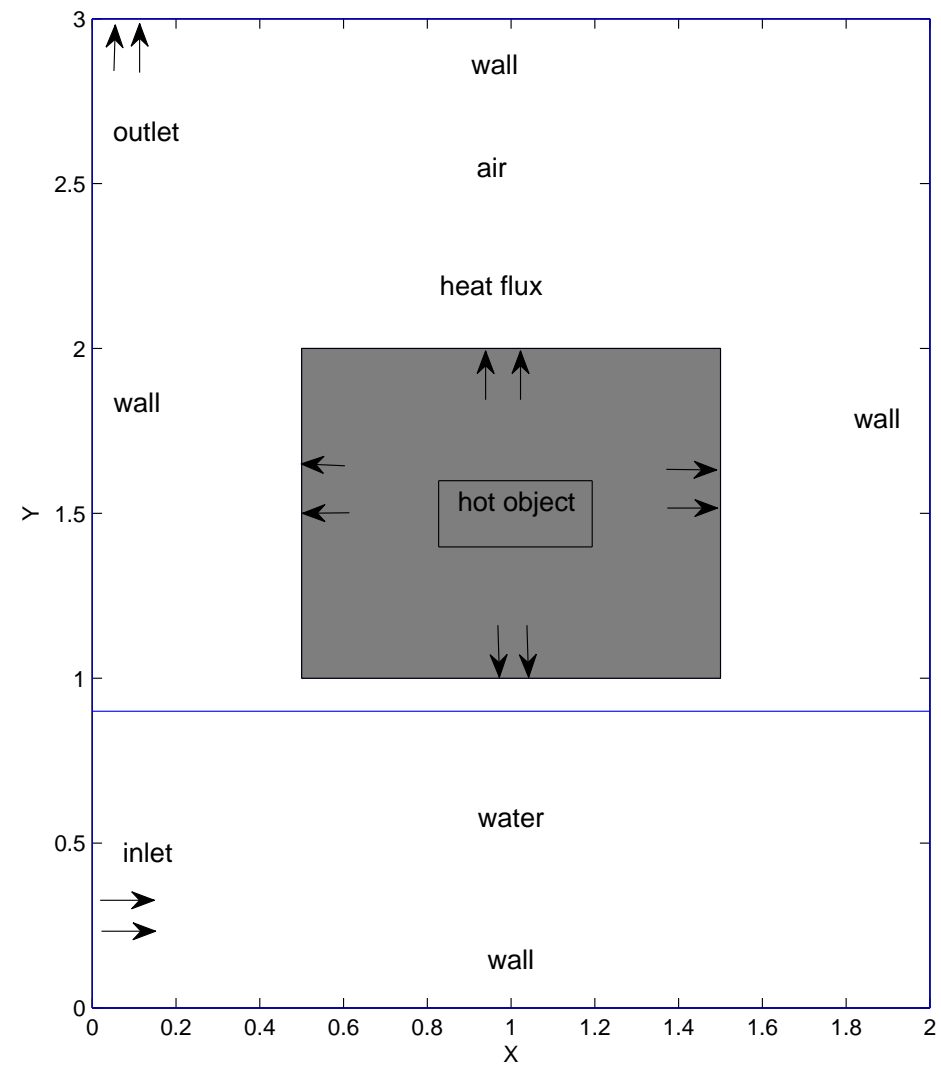

Figure 13: Hot object in air/water cavity: problem configuration. 


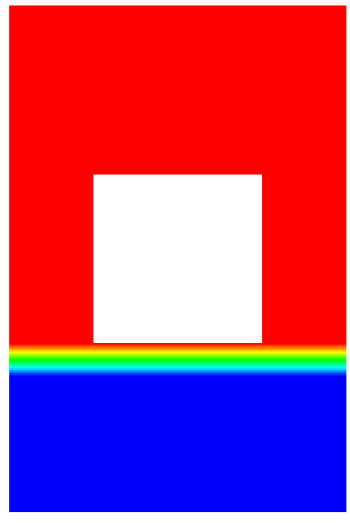

(a)

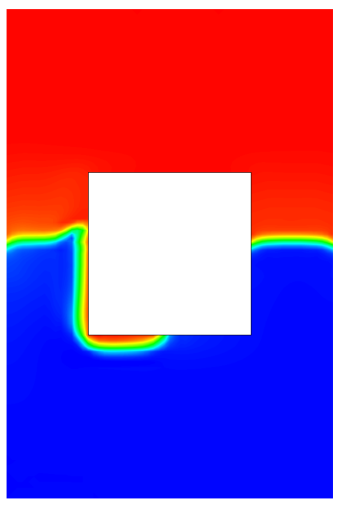

(d)

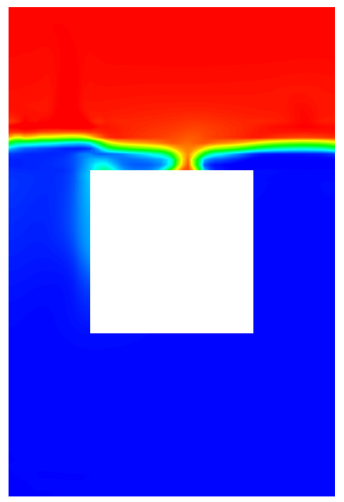

$(\mathrm{g})$

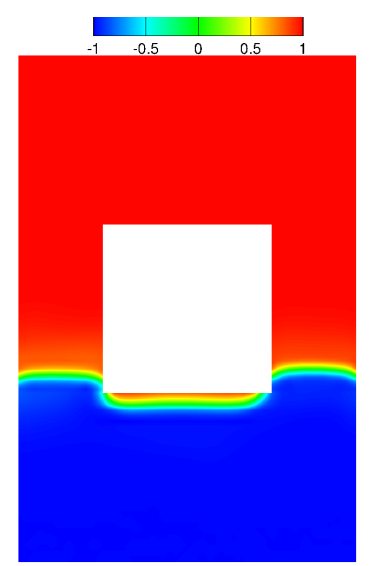

(b)

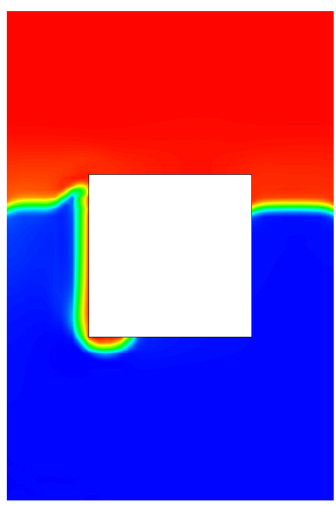

(e)

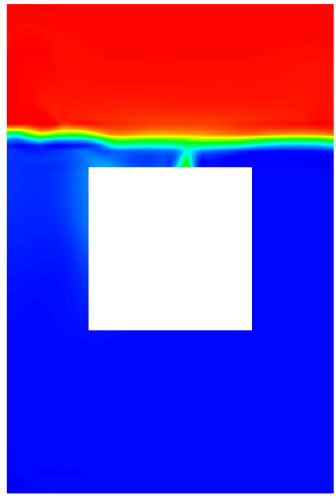

(h)

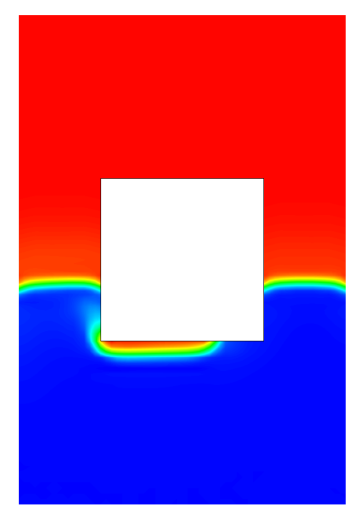

(c)

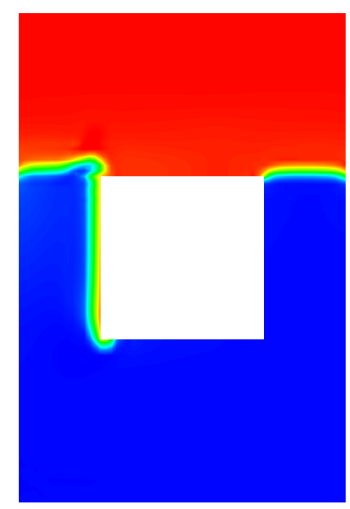

(f)

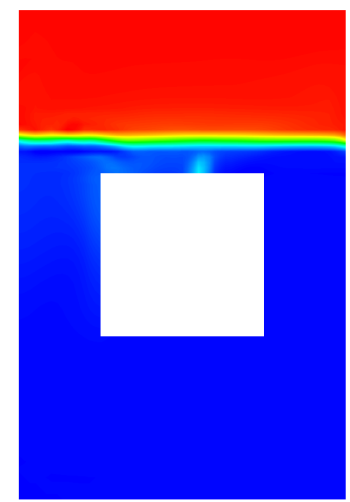

(i)

Figure 14: Phase-field snapshots of air-water interface at time instants on $\mathrm{x}-\mathrm{y}$ plane at $\mathrm{z}=0$ :

(a) $\mathrm{t}=0,(\mathrm{~b}) \mathrm{t}=2.1,(\mathrm{c}) \mathrm{t}=4.1$, (d) $\mathrm{t}=6.1,(\mathrm{e}) \mathrm{t}=8.1$, (f) $\mathrm{t}=10.1,(\mathrm{~g}) \mathrm{t}=11.9$, (h) $\mathrm{t}=12.4$, (i) $\mathrm{t}=12.9$. 


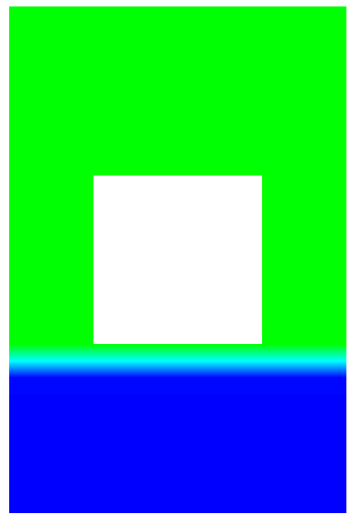

(a)

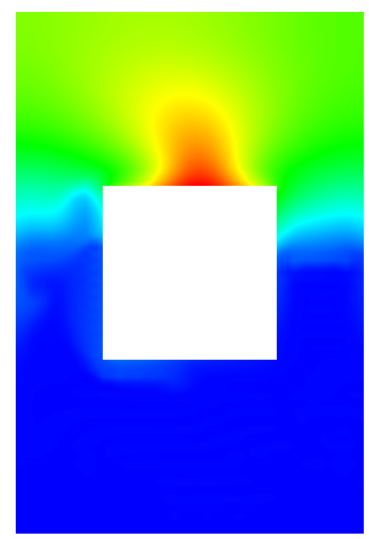

(d)

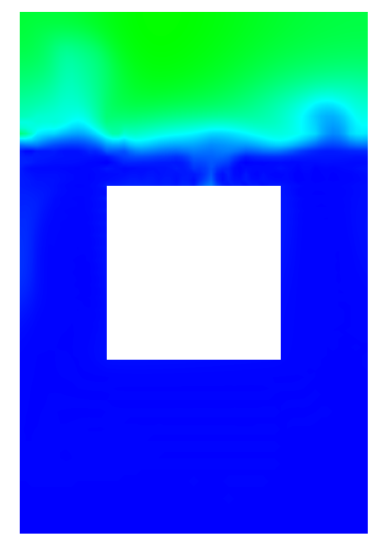

(g)

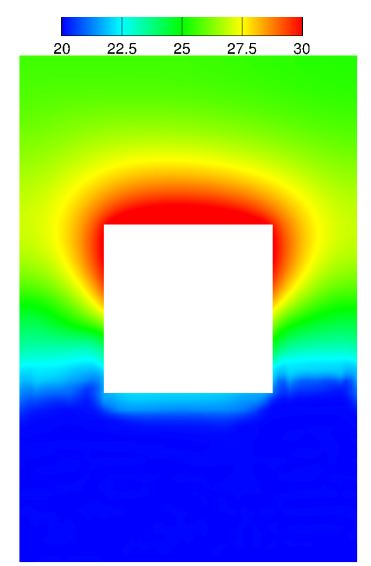

(b)

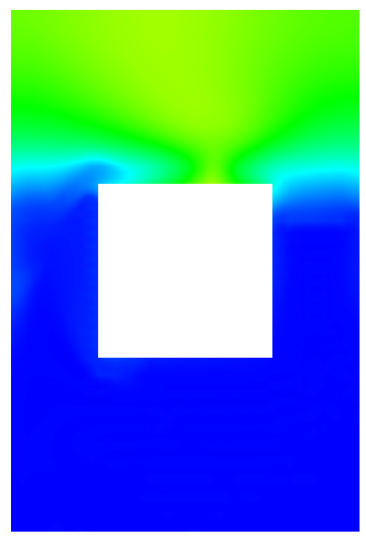

(e)

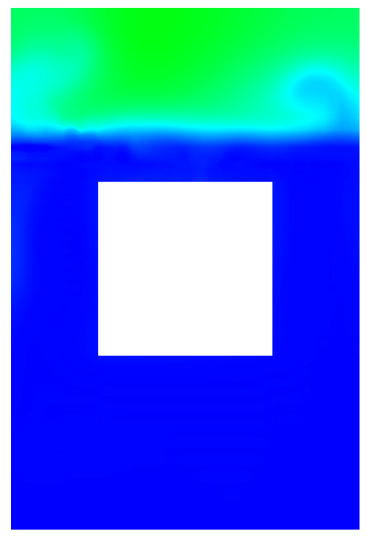

(h)

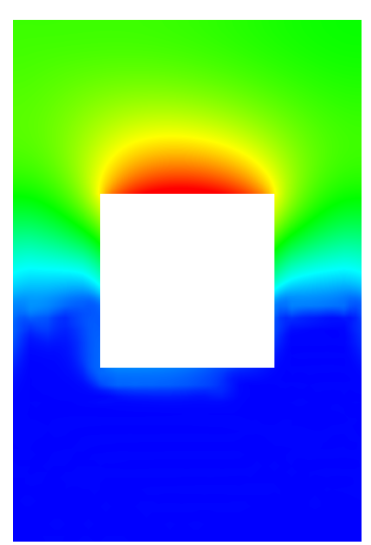

(c)

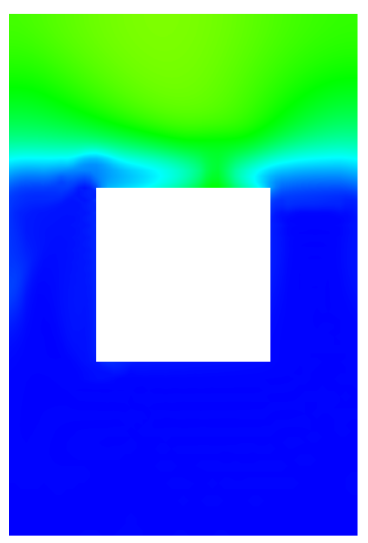

(f)

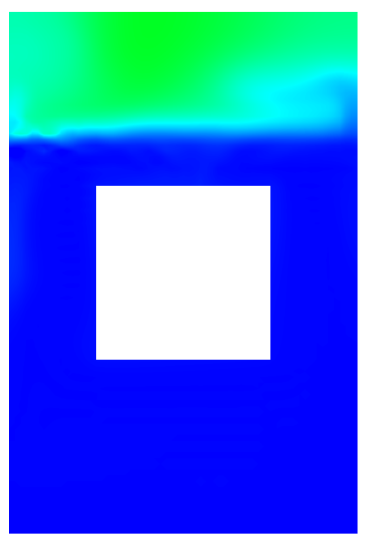

(i)

Figure 15: Snapshots of temperature fields at time instants on $x-y$ plane at $z=0$ : (a) $t=0$, (b) $\mathrm{t}=2.1$, (c) $\mathrm{t}=4.1$, (d) $\mathrm{t}=6.1$, (e) $\mathrm{t}=8.1$, (f) $\mathrm{t}=10.1$, (g) $\mathrm{t}=11.9$, (h) $\mathrm{t}=12.4$, (i) $\mathrm{t}=12.9$. 


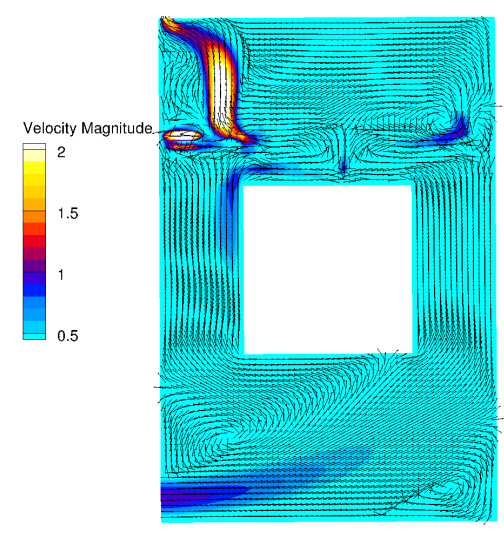

(a)

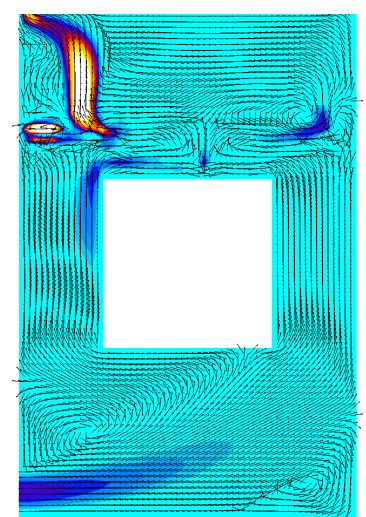

(b)

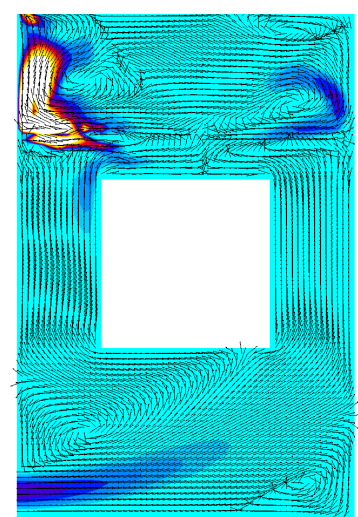

(c)

Figure 16: Snapshots of velocity fields at time instants on $x-y$ plane at $z=0$ : (a) $t=11.9$, (b) $t=12.4$, (c) $t=12.9$. The contour shows the magnitude of the three-dimensional velocity field. The color is intentionally clipped for $|\mathbf{u}|>2$ for better clarity.

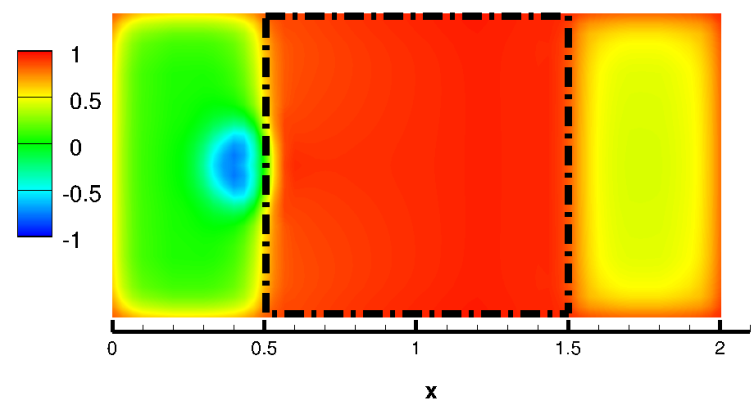

(a)

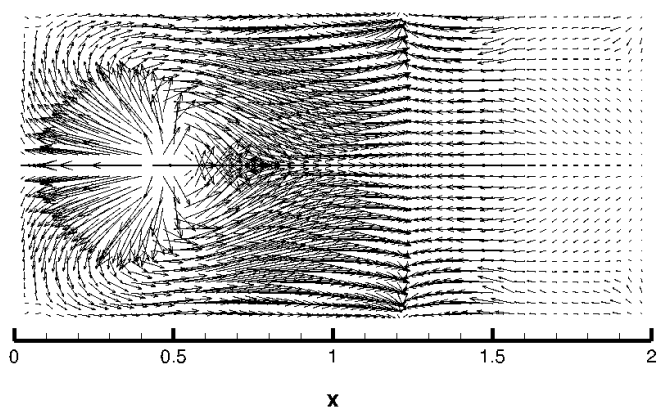

(b)

Figure 17: Snapshots of the phase field and the velocity field on $\mathrm{x}-\mathrm{z}$ plane at $\mathrm{y}=2.05$ and $\mathrm{t}=10.1$. (a) contour plot of the phase field with dash-dot line showing the position of the hot object in X-Z plane; (b) vector plot of the velocity field. 


\section{Summary}

A time-stepping splitting-type numerical method for the two-phase temperature equation is presented. Combined with the algorithm for two-phase flow in [19], an efficient method to simulate two-phase heat transfer problems is developed.

The numerical challenge comes from the variable physical parameters such as density, viscosity, thermal conductivity and specific heat. Standard numerical methods may result in time-dependent coefficient matrices after discretization, which can be time consuming for computation. Here we adopted the strategy of splitting the variable coefficient terms to produce time independent matrices. These coefficient matrices can be computed during preprocessing and this greatly reduces the computation cost. Moreover, this method inherits all the advantages from a splitting-type scheme since all flow variables are completely decoupled.

Numerical convergence tests demonstrate the numerical accuracy of this method quantitatively, i.e., we obtain exponential convergence in space for problems without discontinuities and second-order in time. Benchmark tests of laminar forced convection (single-phase and two-phase) further show that our method is physically accurate for convective heat transfer problem. The simulation of a conjugate heat transfer problem demonstrates that we can handle accurately different modes of heat transfer on a single grid and without iterations between domains. In addition, transient cooling of a hot object in a 3D cavity is carried out. It demonstrates the ability of the method to handle the two-phase heat transfer problem involving a large density ratio and a large viscosity ratio.

In ongoing work we consider additional enhancements of the method. First, we will assume that the surface tension and the individual physical parameters (density, dynamic 
viscosity, specific heat and thermal conductivity) of the fluids are temperature-dependent instead of temperature-independent as in the current method. Second, we will extend our method to include phase change, i.e., phase transition.

\section{Acknowledgement}

We acknowledge the support from ONR through projects N00014-08-1-0080 and N0001414-1-0166 and Sea Grant program in Massachusetts Institute of Technology through project NA10 OAR 4170086. S. D. also wants to acknowledge the support from NSF through project DMS-1318820 and ONR through project N000141110028. High Performance Computing resources were provided by the Office of Naval Research through project N00014-11-1-0598 and project N000141110028.

\section{Appendices}

\section{Appendix A. Phase-field algorithm for incompressible two-phase flow}

This appendix summarizes an algorithm for the incompressible two-phase flow equations (1a)-(40c), together with the boundary conditions (3), (4a) and (4b). The main formulation of this algorithm was developed in [21].

We re-write (1a) into an equivalent but slightly different form,

$$
\rho\left(\frac{\partial \mathbf{u}}{\partial t}+\mathbf{u} \cdot \nabla \mathbf{u}\right)=-\nabla P+\mu \nabla^{2} \mathbf{u}+\nabla \mu \cdot\left(\nabla \mathbf{u}+\nabla \mathbf{u}^{T}\right)-\lambda\left(\nabla^{2} \phi\right) \nabla \phi+\mathbf{f}(\mathbf{x}, t),
$$

where $P=p+\frac{\lambda}{2} \nabla \phi \cdot \nabla \phi$ is an effective pressure, and will also be loosely called pressure. 
We consider how to solve the system of (24), (40b) and (40c), together with the boundary conditions (3), (4a) and (4b). The formulation of the algorithm is summarized below. Given $\left(\mathbf{u}^{n}, P^{n}, \phi^{n}\right)$, we successively solve for $\phi^{n+1}, P^{n+1}$ and $\mathbf{u}^{n+1}$ as follows:

$\underline{\text { For } \phi^{n+1}}$

$$
\begin{aligned}
& \frac{\gamma_{0} \phi^{n+1}-\hat{\phi}}{\Delta t}+\mathbf{u}^{*, n+1} \cdot \nabla \phi^{*, n+1} \\
&=-\lambda \gamma_{1} \nabla^{2}\left[\nabla^{2} \phi^{n+1}-\frac{S}{\eta^{2}}\left(\phi^{n+1}-\phi^{*, n+1}\right)-h\left(\phi^{*, n+1}\right)\right]+g^{n+1}, \\
& \mathbf{n} \cdot \nabla {\left.\left[\nabla^{2} \phi^{n+1}-\frac{S}{\eta^{2}}\left(\phi^{n+1}-\phi^{*, n+1}\right)-h\left(\phi^{*, n+1}\right)\right]\right|_{\partial \Omega}=g_{a}^{n+1}, } \\
&\left.\mathbf{n} \cdot \nabla \phi^{n+1}\right|_{\partial \Omega}=-\frac{1}{\lambda} f_{w}^{\prime}\left(\phi^{*, n+1}\right)+g_{b}^{n+1}
\end{aligned}
$$

$\underline{\text { For } P^{n+1}}$

$$
\begin{gathered}
\frac{\gamma_{0} \tilde{\mathbf{u}}^{n+1}-\hat{\mathbf{u}}}{\Delta t}+\frac{1}{\rho_{0}} \nabla P^{n+1}=-\mathbf{N}\left(\mathbf{u}^{n}\right)+\left(\frac{1}{\rho_{0}}-\frac{1}{\rho^{n+1}}\right) \nabla P^{n}-\frac{\mu^{n+1}}{\rho^{n+1}} \nabla \times \boldsymbol{\omega}^{n} \\
+\frac{1}{\rho^{n+1}} \nabla \mu^{n+1} \cdot \mathbf{D}\left(\mathbf{u}^{n}\right)-\frac{\lambda}{\rho^{n+1}} \nabla^{2} \phi^{n+1} \nabla \phi^{n+1}+\frac{1}{\rho^{n+1}} \mathbf{f}^{n+1}, \\
\nabla \cdot \tilde{\mathbf{u}}^{n+1}=0 \\
\left.\mathbf{n} \cdot \tilde{\mathbf{u}}^{n+1}\right|_{\partial \Omega}=\mathbf{n} \cdot \mathbf{w}^{n+1} .
\end{gathered}
$$

$\underline{\text { For } \mathbf{u}^{n+1}}$

$$
\begin{aligned}
\frac{\gamma_{0} \mathbf{u}^{n+1}-\gamma_{0} \tilde{\mathbf{u}}^{n+1}}{\Delta t} & -\nu_{m} \nabla^{2} \mathbf{u}^{n+1}=-\mathbf{N}\left(\mathbf{u}^{*, n+1}\right)+\mathbf{N}\left(\mathbf{u}^{n}\right)+\nu_{m} \nabla \times \boldsymbol{\omega}^{*, n+1} \\
& +\left(\frac{1}{\rho_{0}}-\frac{1}{\rho^{n+1}}\right) \nabla\left(P^{n+1}-P^{n}\right)-\frac{\mu^{n+1}}{\rho^{n+1}} \nabla \times\left(\boldsymbol{\omega}^{*, n+1}-\boldsymbol{\omega}^{n}\right) \\
& +\frac{1}{\rho^{n+1}} \nabla \mu^{n+1} \cdot\left[\mathbf{D}\left(\mathbf{u}^{*, n+1}\right)-\mathbf{D}\left(\mathbf{u}^{n}\right)\right]
\end{aligned}
$$




$$
\left.\mathbf{u}^{n+1}\right|_{\partial \Omega}=\mathbf{w}^{n+1}
$$

In the above equations, $\tilde{\mathbf{u}}^{n+1}$ is an intermediate velocity, an approximation of $\mathbf{u}^{n+1}$. $\mathbf{N}(\mathbf{u})=\mathbf{u} \cdot \nabla \mathbf{u}$, and $\mathbf{D}(\mathbf{u})=\nabla \mathbf{u}+\nabla \mathbf{u}^{T}$. If $\chi$ denotes a generic variable, $\hat{\chi}$ and $\chi^{*, n+1}$ have the same meanings as those defined in Section 2.2. $\rho^{n+1}$ and $\mu^{n+1}$ are respectively the density and dynamic viscosity at time step $(n+1)$, determined from equation $(2)$ based on $\phi^{n+1}$. The constant $\rho_{0}$ is given by $\rho_{0}=\min \left(\rho_{1}, \rho_{2}\right)$. The parameter $\nu_{m}$ is a chosen constant satisfying $\nu_{m} \geqslant \frac{1}{2}\left(\frac{\mu_{1}}{\rho_{1}}+\frac{\mu_{2}}{\rho_{2}}\right)[19] . S$ is a chosen constant satisfying condition $S \geqslant \eta^{2} \sqrt{\frac{4 \gamma_{0}}{\lambda \gamma_{1} \Delta t}}$.

To implement the above scheme, we reformulate (25a) into two de-coupled Helmholtz type equations (see [21] for details):

$$
\begin{aligned}
& \nabla^{2} \psi^{n+1}-\left(\alpha+\frac{S}{\eta^{2}}\right) \psi^{n+1}=Q \\
& \nabla^{2} \phi^{n+1}+\alpha \phi^{n+1}=\psi^{n+1}
\end{aligned}
$$

where $\psi^{n+1}$ is an auxiliary variable, $Q=\frac{1}{\lambda \gamma_{1}}\left(g^{n+1}-\mathbf{u}^{*, n+1} \cdot \nabla \phi^{*, n+1}+\frac{\hat{\phi}}{\Delta t}\right)+\nabla^{2}\left[h\left(\phi^{*, n+1}\right)-\frac{S}{\eta^{2}} \phi^{*, n+1}\right]$, and $\alpha$ is a constant given by $\alpha=-\frac{S}{2 \eta^{2}}\left(1-\sqrt{1-\frac{4 \gamma_{0}}{\lambda \gamma_{1} \Delta t} \frac{\eta^{4}}{S^{2}}}\right)$. In light of $(29)$ and $(25 \mathrm{c})$, the boundary condition (25b) can be transformed to

$\left.\mathbf{n} \cdot \nabla \psi^{n+1}\right|_{\partial \Omega}=\left.\left(\alpha+\frac{S}{\eta^{2}}\right)\left(-\frac{1}{\lambda} f_{w}^{\prime}\left(\phi^{*, n+1}\right)+g_{b}^{n+1}\right)\right|_{\partial \Omega}+\left.\mathbf{n} \cdot \nabla\left[h\left(\phi^{*, n+1}\right)-\frac{S}{\eta^{2}} \phi^{*, n+1}\right]\right|_{\partial \Omega}+g_{a}^{n+1}$

Let $\varphi \in H^{1}(\Omega)$ denote the test function. By taking the $L^{2}$ inner product between equation 
(28) and $\varphi$, we obtain the weak form about $\psi$,

$$
\begin{aligned}
& \int_{\Omega} \nabla \psi^{n+1} \cdot \nabla \varphi+(\alpha\left.+\frac{S}{\eta^{2}}\right) \int_{\Omega} \psi^{n+1} \varphi \\
&=-\int_{\Omega}\left[g^{n+1}-\mathbf{u}^{*, n+1} \cdot \nabla \phi^{*, n+1}+\frac{\hat{\phi}}{\Delta t}\right] \varphi+\int_{\Omega} \nabla\left[h\left(\phi^{*, n+1}\right)-\frac{S}{\eta^{2}} \phi^{*, n+1}\right] \cdot \nabla \varphi+\int_{\partial \Omega} g_{a}^{n+1} \varphi \\
&+\left(\alpha+\frac{S}{\eta^{2}}\right) \int_{\partial \Omega}\left(-\frac{1}{\lambda} f_{w}^{\prime}\left(\phi^{*, n+1}\right)+g_{b}^{n+1}\right) \varphi, \quad \forall \varphi \in H^{1}(\Omega), \quad(31)
\end{aligned}
$$

where we have used (30). Similarly, one can obtain from (29) the weak form about $\phi^{n+1}$,

$$
\int_{\Omega} \nabla \phi^{n+1} \cdot \nabla \varphi-\alpha \int_{\Omega} \phi^{n+1} \varphi=-\int_{\Omega} \psi^{n+1} \varphi+\int_{\partial \Omega}\left(-\frac{1}{\lambda} f_{w}^{\prime}\left(\phi^{*, n+1}\right)+g_{b}^{n+1}\right) \varphi, \quad \forall \varphi \in H^{1}(\Omega) .
$$

Equations (31) and (32) can be readily discretized using $C^{0}$ spectral elements or finite elements. They are successively solved for $\psi^{n+1}$ and $\phi^{n+1}$ in an un-coupled fashion.

Let $q \in H^{1}(\Omega)$ denote the test function. Take the $L^{2}$-inner product between (26a) and $\nabla q$, and we obtain the following weak form for $P^{n+1}$,

$$
\begin{aligned}
\int_{\Omega} \nabla P^{n+1} \cdot \nabla q= & \rho_{0} \int_{\Omega}\left[\mathbf{G}+\nabla\left(\frac{\mu^{n+1}}{\rho^{n+1}}\right) \times \boldsymbol{\omega}^{n}\right] \cdot \nabla q-\rho_{0} \int_{\partial \Omega} \frac{\mu^{n+1}}{\rho^{n+1}} \mathbf{n} \times \boldsymbol{\omega}^{n} \cdot \nabla q \\
& -\frac{\gamma_{0} \rho_{0}}{\Delta t} \int_{\partial \Omega} \mathbf{n} \cdot \mathbf{w}^{n+1} q, \quad \forall q \in H^{1}(\Omega),
\end{aligned}
$$

where

$$
\mathbf{G}=\frac{1}{\rho^{n+1}}\left[\mathbf{f}^{n+1}-\lambda\left(\psi^{n+1}-\alpha \phi^{n+1}\right) \nabla \phi^{n+1}+\nabla \mu^{n+1} \cdot \mathbf{D}\left(\mathbf{u}^{n}\right)\right]+\frac{\hat{\mathbf{u}}}{\Delta t}-\mathbf{N}\left(\mathbf{u}^{n}\right)+\left(\frac{1}{\rho_{0}}-\frac{1}{\rho^{n+1}}\right) \nabla P^{n}
$$

and $\boldsymbol{\omega}=\nabla \times \mathbf{u}$ denotes the vorticity, and we have used equations (26b) and (26c).

Let $H_{0}^{1}(\Omega)=\left\{v \in H^{1}(\Omega):\left.v\right|_{\partial \Omega}=0\right\}$, and $\varphi \in H_{0}^{1}(\Omega)$ denote the test function. Take the $L^{2}$-inner product between $(27 \mathrm{a})$ and $\varphi$, and note that the intermediate velocity can be substituted by, according to (26a), $\frac{\gamma_{0}}{\Delta t} \tilde{\mathbf{u}}^{n+1}=\mathbf{G}-\frac{\mu^{n+1}}{\rho^{n+1}} \nabla \times \boldsymbol{\omega}^{n}-\frac{1}{\rho_{0}} \nabla P^{n+1}$. We then obtain 
the weak form about $\mathbf{u}^{n+1}$ :

$$
\begin{aligned}
\frac{\gamma_{0}}{\nu_{m} \Delta t} \int_{\Omega} \varphi \mathbf{u}^{n+1}+\int_{\Omega} \nabla \varphi \cdot \nabla \mathbf{u}^{n+1} & =\frac{1}{\nu_{m}} \int_{\Omega}\left[\mathbf{R}+\nabla\left(\frac{\mu^{n+1}}{\rho^{n+1}}\right) \times \boldsymbol{\omega}^{*, n+1}\right] \varphi \\
& -\frac{1}{\nu_{m}} \int_{\Omega}\left(\frac{\mu^{n+1}}{\rho^{n+1}}-\nu_{m}\right) \boldsymbol{\omega}^{*, n+1} \times \nabla \varphi \quad \forall \varphi \in H_{0}^{1}(\Omega) .
\end{aligned}
$$

where

$$
\begin{aligned}
\mathbf{R}=\frac{1}{\rho^{n+1}}\left[\mathbf{f}^{n+1}-\lambda\left(\psi^{n+1}-\alpha \phi^{n+1}\right) \nabla \phi^{n+1}+\nabla \mu^{n+1}\right. & \left.\cdot \mathbf{D}\left(\mathbf{u}^{*, n+1}\right)\right] \\
& +\frac{\hat{\mathbf{u}}}{\Delta t}-\mathbf{N}\left(\mathbf{u}^{*, n+1}\right)-\frac{1}{\rho^{n+1}} \nabla P^{n+1} .
\end{aligned}
$$

The weak forms (33) and (35) can be readily discretized in space with $C^{0}$ spectral elements or finite elements.

The final algorithm consists of: (i) solving (31) and (32) successively for $\psi^{n+1}$ and $\phi^{n+1}$, (ii) solving (33) for $P^{n+1}$, and (iii) solving (35) for $\mathbf{u}^{n+1}$. Note that only constant coefficient matrices are involved in these equations, which can be pre-computed during pre-processing, even though variable density and variable viscosity are involved in the original Navier-Stokes equation.

Finally, as discussed in detail in [21], when the density ratio of the two fluids becomes very large or conversely very small (typically beyond $10^{2}$ or below $10^{-2}$ ) we will slightly modify equation (2) when computing $\rho^{n+1}$ and $\mu^{n+1}$. Specifically, given $\phi^{n+1}$, at large density ratios we compute

$$
\tilde{\phi}^{n+1}= \begin{cases}\phi^{n+1}, & \text { if }\left|\phi^{n+1}\right| \leqslant 1 \\ \operatorname{sign}\left(\phi^{n+1}\right), & \text { if }\left|\phi^{n+1}\right|>1\end{cases}
$$


and then compute $\rho^{n+1}$ and $\mu^{n+1}$ by

$$
\left\{\begin{array}{l}
\rho^{n+1}=\frac{1}{2}\left(\rho_{1}+\rho_{2}\right)+\frac{1}{2}\left(\rho_{1}-\rho_{2}\right) \tilde{\phi}^{n+1} \\
\mu^{n+1}=\frac{1}{2}\left(\mu_{1}+\mu_{2}\right)+\frac{1}{2}\left(\mu_{1}-\mu_{2}\right) \tilde{\phi}^{n+1} .
\end{array}\right.
$$

\section{Appendix B. Non-dimensionalization of variables and governing equations}

Let the superscript in (.)* denote the dimensionalized variable and (.) corresponding to nondimensionalized variable. Below we show how we non-dimensionalize the variables in Section 3.

$$
\left\{\begin{array}{l}
x=\frac{x^{*}}{L}, t=\frac{t^{*}}{L / U_{0}}, \\
p=\frac{p^{*}}{\rho_{1} U_{0}^{2}}, u=\frac{u^{*}}{U_{0}}, T=\frac{T^{*}}{T_{0}} \\
\eta=C_{n}=\frac{\eta^{*}}{L}(\text { Cahn number }), \sigma=\frac{1}{W_{e}}=\frac{\sigma^{*}}{\rho_{1} U_{0}^{2} L}, \lambda=\frac{\lambda^{*}}{\rho_{1} U_{0}^{2} L}=\frac{3}{2 \sqrt{2}} \sigma^{*} \eta^{*} \\
\gamma_{1}=\frac{\gamma_{1}^{*}}{\frac{L}{\left(\rho_{1} U_{0}\right)}}=\frac{W_{e} C_{n}}{P_{e}}, \text { where } P_{e}=\frac{U_{0} \eta^{*} L}{\sigma^{*} \gamma_{1}^{*}}(\text { Peclet number }) \\
\rho(\phi)=\frac{\rho^{*}(\phi)}{\rho_{1}}=\frac{1}{2}\left(1+\frac{\rho_{2}}{\rho_{1}}\right)+\frac{1}{2}\left(1-\frac{\rho_{2}}{\rho_{1}}\right) \phi \\
\mu(\phi)=\frac{1}{R_{e}} \frac{\mu^{*}(\phi)}{\mu_{1}}=\frac{\mu_{1}}{\rho_{1} U_{0} L}\left[\frac{1}{2}\left(1+\frac{\mu_{2}}{\mu_{1}}\right)+\frac{1}{2}\left(1-\frac{\mu_{2}}{\mu_{1}}\right) \phi\right] \\
c(\phi)=\frac{1}{2}\left(1+\frac{c_{2}}{c_{1}}\right)+\frac{1}{2}\left(1-\frac{c_{2}}{c_{1}}\right) \phi \\
k(\phi)=\frac{1}{P_{r} R_{e}} \frac{k(\phi)}{k_{1}}=\frac{k_{1}}{\rho_{1} c_{1} U_{0} L}\left[\frac{1}{2}\left(1+\frac{k_{2}}{k_{1}}\right)+\frac{1}{2}\left(1-\frac{k_{2}}{k_{1}}\right) \phi\right],
\end{array}\right.
$$

where $P_{r}=\frac{\mu_{1}}{\frac{k_{1}}{c_{1}}}$ (Prandtl number) and $W_{e}=\frac{\rho_{1} U_{0}^{2} L}{\sigma^{*}}$ (Weber number). 
Correspondingly, the non-dimensionalized governing equations are given by

$$
\begin{aligned}
& \rho\left(\frac{\partial \mathbf{u}}{\partial t}+\mathbf{u} \cdot \nabla \mathbf{u}\right)=-\nabla p+\nabla \cdot\left[\mu\left(\nabla \mathbf{u}+\nabla \mathbf{u}^{T}\right)\right]-\lambda \nabla \cdot(\nabla \phi \nabla \phi)+\mathbf{f}(\mathbf{x}, t) \\
& \nabla \cdot \mathbf{u}=0 \\
& \frac{\partial \phi}{\partial t}+\mathbf{u} \cdot \nabla \phi=-\lambda \gamma_{1} \nabla^{2}\left[\nabla^{2} \phi-h(\phi)\right]+g(\mathbf{x}, t), \\
& \rho c\left(\frac{\partial T}{\partial t}+\mathbf{u} \cdot \nabla T\right)=\nabla \cdot(k \nabla T)+q_{s}(\mathbf{x}, t)
\end{aligned}
$$

where $h(\phi)=\frac{1}{\eta^{2}} \phi\left(\phi^{2}-1\right)$, and $f=\frac{L}{\rho_{1} U_{0}^{2}} f, g=\frac{L}{U_{0}} g, q_{s}=\frac{L}{\rho_{1} c_{1} U_{0} T_{d}} q_{s}$. The boundary conditions for the velocity, the phase-field and the temperature are non-dimensionalized accordingly.

The system therefore involves several non-dimensional parameters: density ratio $\frac{\rho_{2}}{\rho_{1}}$, dynamic viscosity ratio $\frac{\mu_{2}}{\mu_{1}}$, specific heat ratio $\frac{c_{2}}{c_{1}}$, thermal conductivity ratio $\frac{k_{2}}{k_{1}}$, Cahn number $C_{n}$, Weber number $W_{e}$, Peclet number $P_{e}$, Reynolds number $R_{e}$, Prandtl number $P_{r}$, and contact angle $\theta_{s}$. When the gravity is taken into account, it also involves the Froude number $F_{r}=\frac{U_{0}}{\sqrt{g_{r} L}}$, where $g_{r}$ is the gravitational acceleration. We note that, when the flow variables and physical parameters are non-dimensionalized as given above, the non-dimensional governing equations and the boundary conditions have the same forms as the original dimensional ones.

\section{Appendix C. Initial and boundary conditions for laminar forced convection in a circular pipe simulation}

Below are the initial and boundary conditions for single-phase laminar forced convection: For velocity, Dirichlet boundary condition with $\mathrm{w}=1$ is imposed at inlet. we have the $\partial \mathbf{u} / \partial n=0$ condition imposed. For phase field, Dirichlet for all the boundaries. For temperature, at the outlet we impose Neunmann boundary condition. 
Below are the initial and boundary conditions for two-phase laminar forced convection: For velocity, we use the following fully developed velocity profile at the inlet and also as the initial set up. It can be found in [26]:

$$
\left\{\begin{array}{l}
U_{1}(R)=\frac{u_{1}(r)}{\bar{u}}=\frac{C\left[1-\delta^{2}+\hat{\mu}\left(\delta^{2}-R^{2}\right)\right]}{\delta^{n+3}\left(\hat{\mu_{1}+1}\right)}, 0 \leq R \leq \delta \\
U_{2}(R)=\frac{u_{2}(r)}{\bar{u}}=\frac{C\left[1-R^{2}\right]}{\delta^{n+3}\left(\hat{\left.\mu_{1}+1\right)}\right.}, \delta \leq R \leq 1
\end{array}\right.
$$

where $R=\frac{r}{r_{2}}, \hat{u}=\frac{\mu_{2}}{\mu_{1}}, \delta=\frac{r_{1}}{r_{2}}, C=2$. Boundary condition at outlet is the same as singlephase case. For phase field, profile at inlet is the same as the initial set up as in Eq. (22). Contact-angle boundary conditions are imposed on the pipe walls and outlet with $\theta_{s}=90$. For temperature, boundary condition at the outlet is the same as single-phase case.

\section{Appendix D. Laminar forced convection for a single-phase flow in a rectangular duct}

Here we present the result of simulating laminar convection for a hydrodynamically fully developed and thermally developing single-phase flow in a rectangular duct. We use the same initial conditions, boundary conditions and physical paramters (density, dynamic viscosity, thermal conductivity and specific heat) as we do for simulating a single-phase flow in a pipe (see Appendix C). We discretize the domain with 3000 hexahedron elements (10 in $\mathrm{x}$ direction, 10 in y direction and 30 in z direction). An element of order 3 has been used for all the elements. A time step size $\Delta t=10^{-3}$ has been used in the simulation.

We simulate five different temperature boundary conditions with one or more walls heated. Then we compare the Nusselt numbers caculated from our simulation to the empirical values given in [24]. Table 5 shows that there is a good agreement between the Nusselt numbers from our simulation and those give in [24]. 
Table 5: Nusselt numbers from simulation and the handbook [24] for fully developed laminar flow in rectangular ducts with one wall or more walls heating.

\begin{tabular}{|l|l|l|l|l|l|}
\hline Boundaries & all walls & one wall & Two adjacent & Three walls & Two opposite \\
& Dirichlet & Neumann & walls Neumann & Neumann & walls Neumann \\
\hline Simulation & 2.970 & 3.018 & 3.703 & 2.437 & 2.375 \\
\hline Handbook [24] & 2.912 & 3.115 & 4.030 & 2.532 & 2.402 \\
\hline
\end{tabular}

\section{Appendix E. Initial and boundary conditions for transient cooling of a hot object} in a $3 \mathrm{D}$ cavity

Position for inlet: $x=0,(y, z): 0 \leq y \leq 0.3,-0.5 \leq z \leq 0.5$. Center for inlet: $x=0$, $y=0.15, z=0$. Position for outlet: $y=3,(x, z): 0 \leq x \leq 0.1,-0.5 \leq z \leq 0.5$. Center for outlet: $x=0.05, y=3, z=0$.

Boundary conditions for the velocity field $\mathbf{u}(u, v, w)$ are as follows. Velocity profile at inlet:

$$
u=V_{0} *\left(1.0-\left(\left(y-Y_{I N}\right) / R_{0}\right)^{2}\right) *\left(1.0-\left(\left(z-Z_{I N}\right) / R_{1}\right)^{2}\right),
$$

where $Y_{I N}=0.15$ and $Z_{I N}=0$ are the position of the center of the inlet in y and z direction, $R_{0}=0.15$ and $R_{1}=0.5$ are the half width of the inlet in $\mathrm{y}$ and $\mathrm{z}$ direction and $V_{0}=1.0$. Other two components of velocity $\mathrm{v}$ and $\mathrm{w}$ are zero. The boundary conditions for all velocity components at the outlet are $\partial \mathbf{u} / \partial n=0$. No slip boundary condition is imposed on the walls of the cavity and the hot object.

Boundary conditions for temperature field are as follows. Temperature is known at the 
inlet. We impose heat flux boundary conditions on the walls of the cavity (including outlet) and on the walls of the hot object.

Below we give the initial set up for the velocity profile at the inlet in the simulation of transient cooling of a hot object in a 3D cavity:

$u=V_{0}\left(1-\left(\left(y-Y_{I N}\right) / R_{0}\right)^{2}\right)\left(1-\left(\left(z-Z_{I N}\right) / R_{1}\right)^{2}\right)\left(1-\operatorname{step}\left(y,\left(Y_{I N}+R_{0}\right)\right)\right)\left(1-\operatorname{step}\left(x, X_{0}\right)\right)$, where $V_{0}, Y_{I N}, Z_{I N}, R_{0}$ and $R_{1}$ are the same as above and $X_{0}=1.6$. Other two components of velocity $\mathrm{v}$ and $\mathrm{w}$ are zero. The $\operatorname{step}(\mathrm{x}, \mathrm{y})$ is the step function as follows:

$$
\operatorname{step}(x, y)=\left\{\begin{array}{l}
0, \text { if } x<=y \\
1, \text { if } x>y
\end{array} .\right.
$$




\section{References}

[1] G. Caginalp, X. Chen, Phase field equations in the singular limit of sharp interface problems, in: In On the Evolution of Phase Boundaries of the IMA Volumes in Mathematics and its Applications, vol. 43, Springer, New York, 1992, pp. 1-27.

[2] Y. C. Chang, T. Y. Hou, B. Merriman, S. Osher, A level set formulation of Eulerian interface capturing methods for incompressible fluid flows, Journal of Computational Physics 124 (2) (1996) 449-464.

[3] S. O. Unverdi, G. Tryggvason, A front-tracking method for viscous, incompressible, multi-fluid flows, Journal of Computational Physics 100 (1) (1992) 25-37.

[4] F. Boyer, A theoretical and numerical model for the study of incompressible mixture flows, Computers \& Fluids 31 (1) (2002) 41-68.

[5] C. W. Hirt, B. D. Nichols, Volume of fluid (VOF) method for the dynamics of free boundaries, Journal of Computational Physics 39 (1) (1981) 201-225.

[6] V. Cristini, J. Blawzdziewicz, M. Loewenberg, Drop breakup in three-dimensional viscous flows, Physics of Fluids 10 (1998) 1781-1783.

[7] H. H. Hu, N. A. Patankar, M. Y. Zhu, Direct numerical simulations of fluid-solid systems using the arbitrary Lagrangian-Eulerian technique, Journal of Computational Physics 169 (2) (2001) 427-462.

[8] J. J. Monaghan, A. Kocharyan, SPH simulation of multi-phase flow, Computer Physics Communications 87 (1-2) (1995) 225-235. 
[9] C. F. Naa, Three-dimensional Smoothed Particle Hydrodynamics simulation for liquid metal solidification process, ArXiv preprint ArXiv:1309.4234, 2013.

[10] A. Valizadeh, M. Shafieefar, J. J. Monaghan, S. A. A. Salehi Neyshaboori, Modeling two-phase flows using SPH method, Journal of Applied Science 8 (2008) 3817-3826.

[11] D. Gerlach, G. Tomar, G. Biswas, F. Durst, Comparison of volume-of-fluid methods for surface tension-dominant two-phase flows, International Journal of Heat and Mass Transfer 49 (3-4) (2006) 740-754.

[12] C. S. Wu, D. L. Young, H. C. Wu, Simulations of multidimensional interfacial flows by an improved volume-of-fluid method, International Journal of Heat and Mass Transfer $60(2013) 739-755$.

[13] Y. Tsui, S. Lin, Y. Lai, F. Wu, Phase change calculations for film boiling flows, International Journal of Heat and Mass Transfer 70 (2014) 745-757.

[14] I. Chakraborty, G. Biswas, P. S. Ghoshdastidar, A coupled level-set and volume-of-fluid method for the buoyant rise of gas bubbles in liquids, International Journal of Heat and Mass Transfer 58 (1-2) (2013) 240-259.

[15] B. Ray, G. Biswas, A. Sharma, Bubble pinch-off and scaling during liquid drop impact on liquid pool, Physics of Fluids 24 (2012) 082108-1 - 082018-11.

[16] A. Criscione, D. Kintea, Z. Tukovic, S. Jakirlic, I. V. Roisman, C. Tropea, Crystallization of supercooled water: A level-set-based modeling of the dendrite tip velocity, International Journal of Heat and Mass Transfer 66 (2013) 830-837. 
[17] D. Jacqmin, Contact-line dynamics of a diffuse fluid interface, Journal of Fluid Mechanics 402 (2000) 57-88.

[18] P. Yue, J. Feng, C. Liu, J. Shen, A diffuse-interface method for simulating two-phase flow of complex fluids, Journal of Fluid Mechanics 515 (2004) 293-317.

[19] S. Dong, On imposing dynamic contact-angle boundary conditions for wall-bounded liquid-gas flows, Computer Methods in Applied Mechanics and Engineering 247-248 (2012) 179-200.

[20] G. E. Karniadakis, S. J. Sherwin, Spectral/hp element methods for computational fluid dynamics, 2nd Edition, Oxford University Press, Oxford, 2005.

[21] S. Dong, J. Shen, A time-stepping scheme involving constant coefficient matrices for phase field simulations of two-phase incompressible flows with large density ratios, Journal of Computational Physics 231 (2012) 5788-5804.

[22] D. Gottlieb, S. A. Orszag, Numerical analysis of spectral methods: theory and applications, 1st Edition, Capital City Press, Montpelier, 1997.

[23] V. E. Badalassi, H. D. Ceniceros, S. Banerjee, Computation of multiphase systems with phase field models, Journal of Computational Physics 190 (2) (2003) 371-397.

[24] W. M. Rohsenow, Handbook of Heat Transfer, McGraw-Hill, New York, 1998.

[25] D. Jacqmin, Calculation of two-phase Navier-Stokes flows using phase-field modeling, Journal of Computational Physics 155 (1) (1999) 96-127. 
[26] E. Nogueira, R. Cotta, R. Janeiro, Heat transfer solutions in laminar co-current flow of immiscible liquids, Warme-und Stoffubertragung 25 (1990) 361-367.

[27] Y. Sun, I. S. Wichman, On transient heat conduction in a one-dimensional composite slab, International Journal of Heat and Mass Transfer 47 (6-7) (2004) 1555-1559.

[28] F. de Monte, Transient heat conduction in one-dimensional composite slab. A 'natural' analytic approach, International Journal of Heat and Mass Transfer 43 (19) (2000) $3607-3619$. 